\title{
LION: A dynamic computer model for the low-latitude ionosphere
}

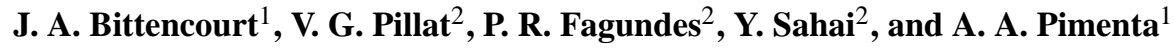 \\ ${ }^{1}$ Instituto Nacional de Pesquisas Espaciais (INPE), C. P. 515, São José dos Campos, SP, Brazil \\ ${ }^{2}$ Universidade do Vale do Paraíba (UNIVAP), Av. Shishima Hifumi, 2911, Urbanova, São José dos Campos, SP, Brazil
}

Received: 25 April 2007 - Revised: 28 September 2007 - Accepted: 30 October 2007 - Published: 29 November 2007

\begin{abstract}
A realistic fully time-dependent computer model, denominated LION (Low-latitude Ionospheric) model, that simulates the dynamic behavior of the low-latitude ionosphere is presented. The time evolution and spatial distribution of the ionospheric particle densities and velocities are computed by numerically solving the time-dependent, coupled, nonlinear system of continuity and momentum equations for the ions $\mathrm{O}^{+}, \mathrm{O}_{2}^{+}, \mathrm{NO}^{+}, \mathrm{N}_{2}^{+}$and $\mathrm{N}^{+}$, taking into account photoionization of the atmospheric species by the solar extreme ultraviolet radiation, chemical and ionic production and loss reactions, and plasma transport processes, including the ionospheric effects of thermospheric neutral winds, plasma diffusion and electromagnetic $\boldsymbol{E} \times \boldsymbol{B}$ plasma drifts. The Earth's magnetic field is represented by a tilted centered magnetic dipole. This set of coupled nonlinear equations is solved along a given magnetic field line in a Lagrangian frame of reference moving vertically, in the magnetic meridian plane, with the electromagnetic $\boldsymbol{E} \times \boldsymbol{B}$ plasma drift velocity. The spatial and time distribution of the thermospheric neutral wind velocities and the pattern of the electromagnetic drifts are taken as known quantities, given through specified analytical or empirical models. The model simulation results are presented in the form of computer-generated color maps and reproduce the typical ionization distribution and time evolution normally observed in the low-latitude ionosphere, including details of the equatorial Appleton anomaly dynamics. The specific effects on the ionosphere due to changes in the thermospheric neutral winds and the electromagnetic plasma drifts can be investigated using different wind and drift models, including the important longitudinal effects associated with magnetic declination dependence and latitudinal separation between geographic and geomagnetic equators. The model runs in a normal personal computer (PC) and generates color maps illustrating the typical behavior of the
\end{abstract}

Correspondence to: J. A. Bittencourt

(jabittencourt@hotmail.com) low-latitude ionosphere for a given longitudinal region, for different seasons, geophysical conditions and solar activity, at each instant of time, showing the time evolution of the lowlatitude ionosphere, between about $20^{\circ}$ north and south of the magnetic equator. This paper presents a detailed description of the mathematical model and illustrative computer results.

Keywords. Ionosphere (Equatorial Ionosphere, Modeling and forecasting)

\section{Introduction}

The distribution of ionization in the low-latitude ionosphere is characterized by the presence of the so-called Appleton anomaly or equatorial ionospheric anomaly. Since the ionospheric ionization is produced as the result of photoionization of the atmospheric species by the solar extreme ultraviolet (XUV) radiation, a maximum of ionization would be expected around the sub-solar point in the low latitudinal regions, where the ionization production is a maximum. However, when measured values of the electron number density at the F2-peak are plotted as a function of magnetic latitude, for a given longitude and local time, a curve is obtained which shows a minimum (trough) over the magnetic dip equator, with two maxima (crests) at dip latitudes which may vary between $10^{\circ}$ to $20^{\circ}$ north and south of the dip equator, depending on local time and season. This anomalous ionization distribution extends into the topside ionosphere where it gradually diminishes. At the magnetic equator the F2-peak height is a maximum and the peak electron density is typically about 20 to 50 percent less than at the crests.

This equatorial ionospheric anomaly was first recognized by Appleton (1946) and has since been investigated by many workers. It shows rather different features depending on longitude, local time, season and period of the sunspot cycle. The diurnal development of the equatorial ionospheric

Published by Copernicus Publications on behalf of the European Geosciences Union. 
anomaly has been studied in some detail for both sunspot maximum and sunspot minimum conditions (Rao, 1962; Lyon and Thomas, 1963; Thomas, 1968; Rastogi, 1966; Rush et al., 1969). Lyon and Thomas (1963) analyzed solar maximum ionosonde data, and showed that the anomaly is enhanced just after sunset, for several hours, and it exists throughout the night and sometimes until sunrise. There is also considerable longitudinal variation in the development and decay of the anomaly. It shows marked differences in the various longitudinal sectors, depending on the corresponding magnetic declination of each sector. A latitudinal asymmetry in the electron density, as well as in the F2-peak height, at the north-south crests has also been observed, and it shows different behavior in the various longitudinal sectors, at the same local time.

This anomalous ionization distribution at low latitudes has been explained in terms of plasma transport processes that move the ionization to regions other than that of its production. It has been investigated theoretically over the past several years by many researchers (e.g. Martyn, 1953; Bramley and Peart, 1965; Hanson and Moffett, 1966; Bramley and Young, 1968; Baxter and Kendall, 1968; Abbur-Robb and Windle, 1969; Sterling et al., 1969; Anderson, 1973a, b; Bittencourt and Tinsley, 1976; Bittencourt et al., 1976, Bittencourt, 1996). The transport processes affecting the ionization distribution in the low-latitude ionosphere are plasma diffusion, electromagnetic plasma drifts, and thermospheric neutral wind drag caused by the meridional and zonal global pressure gradients. A review on the electrodynamics of the equatorial ionosphere-thermosphere system was recently presented by Abdu (2005).

\section{Physical processes}

The physical processes involving production, loss, and transport of ionization in the low-latitude ionosphere have different degrees of importance, depending on the altitude region and the local time under consideration. The ionospheric sources of ionization include both electromagnetic and corpuscular radiations. Because of magnetic shielding effects, corpuscular radiation is important only at high latitudes as an ionizing source. Since in the ionosphere the principal neutral constituent is atomic oxygen, at least $13.6 \mathrm{eV}$ is required for each ion-electron pair created and this energy can be supplied by the solar extreme ultraviolet (XUV) radiation with wavelengths shorter than $91.1 \mathrm{~nm}$. The ionization produced in a given volume of the ionosphere may leave it either by recombining or by moving outside it. The ion and neutral species, and the electrons may interact as a result of chemical and ionic reactions such as electron-ion recombination, involving electrons and either atomic or molecular positive ions, or as ion-ion recombination, involving positive and negative ions. This second loss mechanism is small compared to the first one, since few negative ions exist at F-region altitudes due to a low production rate and loss by photodetachment.

In the F1-region and below, a photochemical equilibrium condition exists during the daytime, since the recombination time constant is sufficiently small and transport processes are relatively unimportant. The recombination time constant increases with altitude, whereas the time constant for loss by diffusion $\left(\tau_{D}\right)$ decreases with increasing altitude. In the F2region, due to the longer recombination lifetime and smaller diffusion lifetime, transport of ionization plays a dominant role. Laboratory and ionospheric measurements of the recombination rates appropriate to the F2-region indicate that the lifetime of an ion-electron pair is about one to two hours (Ferguson, 1969). At an altitude where these recombination time constants are approximately equal, both processes are comparable and, consequently, the electron density as a function of altitude reaches its maximum at approximately this altitude.

The three relevant transport processes that move the ionization to regions other than that of its formation are plasma diffusion along the magnetic field lines, electromagnetic $\boldsymbol{E} \times \boldsymbol{B}$ plasma drifts, which transport the ionization perpendicularly to the magnetic field lines, and thermospheric neutral winds, which drag the ionization in the direction of the wind component along the field line. The plasma drift due to an east-west electric field moves the low-latitude ionospheric ionization perpendicularly to the magnetic field lines, in the well-known fountain effect. This transport process, combined with plasma diffusion along the magnetic field lines, caused by gravity and pressure gradients, produces a symmetrical ionization distribution about the magnetic dip equator. Two crests of plasma concentration are generated around $\pm 15^{\circ}$ (north-south) on either side of the magnetic dip equator. The latitudinal position of these ionization crests vary with local time and season, depending on the time variation of the $\boldsymbol{E} \times \boldsymbol{B}$ plasma drift, as well as with longitude. Hanson and Moffett (1966) illustrated the plasma flow associated with the equatorial fountain effect as a result of the combined effects of $\boldsymbol{E} \times \boldsymbol{B}$ electrodynamic plasma lifting across the magnetic field lines and plasma diffusion along the field lines. The third process is transport due to thermospheric neutral winds. The neutral-ion collisional drag transports the ionization along the magnetic field lines, in the direction of the wind component along the field, producing an interhemisphere transport of ionization at the same time that it moves the ionization upward in the upwind side and downward in the downwind side of the magnetic field line, into regions of different recombination rates. This process results in an asymmetrical ionization distribution about the magnetic dip equator, with unequal values of the electron densities and heights of the F2-peak at the ionization crests around $\pm 15^{\circ}$ either side of the magnetic equator. The ionospheric plasma vertical drift produced by a horizontal thermospheric neutral wind at low latitudes was illustrated schematically in Bittencourt and Sahai (1978). 
At low latitudes these transport processes are greatly dependent on the geometry of the magnetic field lines at a particular region. The longitudinal variations of the magnetic declination and of the latitudinal separation between the geographic and the geomagnetic equators play important roles in the drift and wind effects on the ionospheric plasma. For this purpose it very convenient to separate the tropical ionosphere into three longitudinal sectors (Bittencourt et al., 1976, 1992), based on the value of the magnetic declination at a given longitude, namely:

(a) Atlantic Sector, from $-65^{\circ} \mathrm{W}$ to $0^{\circ}$, where the magnetic declination is west and takes its maximum value (about $20^{\circ} \mathrm{W}$ ) at the magnetic equator.

(b) Indian Sector, from $0^{\circ}$ to $150^{\circ} \mathrm{E}$, where the magnetic declination is everywhere near zero at the magnetic equator.

(c) Pacific Sector, from $150^{\circ} \mathrm{E}$ to $-65^{\circ} \mathrm{W}$, where the magnetic declination is close to $10^{\circ} \mathrm{E}$ at the magnetic equator.

The great advantage of using this classification of longitudinal sectors, in terms of magnetic declination, is that it allows us to separate the specific ionospheric effects of the zonal and meridional thermospheric wind components, as well as the effects associated with the seasonal dependences of the vertical electromagnetic plasma drift velocities.

\section{Basic transport equations}

The appropriate equations governing the spatial and time distribution of the electron and ion densities in the ionosphere are the time-dependent continuity equation, the momentum conservation equation and the energy conservation equation for each charged particle species. The continuum approximation to the Boltzmann equation holds under the assumption that collisions between the particles are so frequent that the ions and electrons can both be treated as fluids. Normally, this assumption is very well justified for thermal particles in the ionosphere. Above about $600 \mathrm{~km}$, in the exosphere, the neutral particles move in balistic orbits and suffer few collisions. However, the plasma fluid approximation is still applicable to even higher altitudes because of the large Coulomb cross sections.

\subsection{The plasma continuity equation}

The continuity equation relates the rate of change in the particle number density to the rate of production and loss per unit volume, and to the divergence of the particle flux. If $n_{i}$ and $\boldsymbol{v}_{i}$ denote the number density and the macroscopic (local average) velocity of the $i$ th ion species, respectively, then the quantity $n_{i} \boldsymbol{v}_{i}$ represents the flux of this charged particle species and its divergence gives the resulting loss rate per unit volume, due to transport. For the $i$ th ion species, the continuity equation can be expressed as (Bittencourt, 2004)

$\frac{\partial n_{i}}{\partial t}+\nabla \cdot\left(n_{i} \boldsymbol{v}_{i}\right)=P_{i}-L_{i}$

where $P_{i}$ and $L_{i}$ are the production and loss rates per unit volume, respectively. The macroscopic charge neutrality plasma condition requires that the electron number density be given by

$n_{e}=\sum_{i} n_{i}$

where only singly charged positive ions are considered, since negative ions are scarce in the ionosphere.

The charged particles in the low-latitude ionosphere may have a common drift velocity due to an external electrostatic field $\boldsymbol{E}$, not parallel to the geomagnetic induction field $\boldsymbol{B}$, such as that associated with the wind-driven dynamo current system of the E-region, which is very effective in transporting ionization across the magnetic field lines, as well as a diffusion velocity along the magnetic field lines arising from non-electromagnetic forces, namely those due to gravitation, pressure gradients and collisions. Although nonelectromagnetic forces, like gravity and pressure gradients, are able to transport ionization across the magnetic field lines, the associated drifts are usually much weaker than the electromagnetic drift.

For calculation purposes it is convenient to separate the particle macroscopic velocity $\boldsymbol{v}_{i}$ into components parallel and perpendicular to the local magnetic field line,

$\boldsymbol{v}_{i}=\boldsymbol{v}_{i \|}+\boldsymbol{v}_{\perp}$

where $\boldsymbol{v}_{\perp}$ corresponds to the electromagnetic $\boldsymbol{E} \times \boldsymbol{B} / B^{2}$ plasma drift velocity. In addition, the natural frame of reference for expressing the charged particle motion is a coordinate system moving with the plasma drift velocity $\boldsymbol{v}_{\perp}$ with respect to a fixed Earth-centered system. The divergence of the plasma flux perpendicular to the magnetic field can be separated into two parts

$\boldsymbol{\nabla} \cdot\left(n_{i} \boldsymbol{v}_{\perp}\right)=\boldsymbol{v}_{\perp} \cdot \nabla n_{i}+n_{i} \boldsymbol{\nabla} \cdot \boldsymbol{v}_{\perp}$

Adding the advective part to $\partial n_{i} / \partial t$ gives

$\frac{D n_{i}}{D t}=\frac{\partial n_{i}}{\partial t}+\boldsymbol{v}_{\perp} \cdot \nabla n_{i}$

which is the total rate of change in the particle number density in a frame of reference moving with the plasma drift velocity $\boldsymbol{v}_{\perp}$.

The great advantage of using this Lagrangian reference frame is that in this system all plasma motions appear to be field aligned. This approach was first used by Moffett and Hanson (1965) in solving the time-dependent electron continuity equation including the effects of diffusion, plasma drift, 
production, and loss. Therefore, in the drifting coordinate system, the continuity equation for each ion species becomes

$$
\frac{D n_{i}}{D t}+\nabla \cdot\left(n_{i} \boldsymbol{v}_{i \|}\right)+n_{i} \boldsymbol{\nabla} \cdot \boldsymbol{v}_{\perp}=P_{i}-L_{i}
$$

An expression for the flux parallel to $\boldsymbol{B}$, for each ion constituent, $n_{i} \boldsymbol{v}_{i \|}$, is derived in the next section starting from the equations of motion for the electrons and ions. The divergence term involving the plasma drift velocity, as well as the production and loss terms, will be considered subsequently.

\subsection{The Equation of motion}

The forces acting on the ionospheric plasma include gravitational, collisional and pressure gradient forces, as well as electric and magnetic forces. The equation of motion for each ion species can be expressed as (Bittencourt, 2004)

$$
\begin{aligned}
& m_{i}\left(\frac{\partial \boldsymbol{v}_{i}}{\partial t}+\left(\boldsymbol{v}_{i} \cdot \nabla\right) \boldsymbol{v}_{i}\right)=e\left(\boldsymbol{E}+\boldsymbol{v}_{i} \times \boldsymbol{B}\right)+m_{i} \boldsymbol{g}- \\
& \frac{1}{n_{i}} \nabla\left(n_{i} k T_{i}\right)-\sum_{n} m_{i} v_{i n}\left(\boldsymbol{v}_{i}-\boldsymbol{u}\right)-\sum_{j}^{\prime} m_{i} v_{i j}\left(\boldsymbol{v}_{i}-\boldsymbol{v}_{j}\right)
\end{aligned}
$$

and for the electrons,

$m_{e}\left(\frac{\partial \boldsymbol{v}_{e}}{\partial t}+\left(\boldsymbol{v}_{e} \cdot \nabla\right) \boldsymbol{v}_{e}\right)=-e\left(\boldsymbol{E}+\boldsymbol{v}_{e} \times \boldsymbol{B}\right)-\frac{1}{n_{e}} \nabla\left(n_{e} k T_{e}\right)$

where it is assumed that, in the equation for the electrons, both the gravitational and the collisional terms are very small compared to the remaining terms and can be neglected (Rishbeth and Garriot, 1969). Also, a scalar pressure $\left(p_{\alpha}=n_{\alpha} k T_{\alpha}\right.$, where $\alpha=e, i$ ) replaces the stress tensor, since the velocity distribution function is isotropic. Subscripts $e, i$, and $n$ refer to electrons, ions, and neutrals, respectively. Mass and temperature are represented by $m$ and $T$, the acceleration due to gravity is $\boldsymbol{g}$, e denotes the electronic charge, $k$ is Boltzmann's constant, $\boldsymbol{u}$ is the thermospheric neutral wind velocity, and $v_{\text {in }}$ and $v_{i j}$ are the effective collision frequencies between ions and neutrals, and ions and ions, respectively.

The acceleration term on the left-hand side of the equation of motion for both electrons and ions (in the moving reference frame) can be set equal to zero, being generally negligible for the large scale motions that constitute the main interest here. However, they might not be negligible for smallscale wavelike motions. Taking the component of Eq. (8) parallel to the magnetic field lines, the polarization electric field generated between electrons and ions is obtained as

$\boldsymbol{E}_{\|}=-\frac{1}{e n_{e}} \nabla_{\|}\left(n_{e} k T_{e}\right)$

Adopting the convention previously used by Kendall (1962), we can write

$\nabla_{\|}=\widehat{t} \frac{\partial}{\partial s}$ where $\hat{\boldsymbol{t}}$ represents a unit vector along the magnetic field line, defined such that for points in the Northern Hemisphere it is above the horizontal and towards the geomagnetic equator, and $\mathrm{s}$ is the arc length along the field line measured in the same sense as $\hat{\boldsymbol{t}}$. Since the cyclotron frequency greatly exceeds the collision frequency with neutrals throughout the F-region, the non-electromagnetic forces will transport the ionization essentially along the field lines. Thus, taking the component of Eq. (7) parallel to the magnetic field, using the expression in Eq. (9) and rearranging, we obtain

$$
\begin{aligned}
& \boldsymbol{v}_{i \|}=\frac{1}{m_{i}\left(\sum_{n} v_{i n}+\sum_{j}^{\prime} v_{i j}\right)}\left[-m_{i} g \sin I-\right. \\
& k \frac{\partial}{\partial s}\left(T_{e}+T_{i}\right)-\frac{k T_{e}}{n_{e}} \frac{\partial n_{e}}{\partial s}-\frac{k T_{i}}{n_{i}} \frac{\partial n_{i}}{\partial s} \\
& \left.+\sum_{n} m_{i} v_{i n}\left|\boldsymbol{u}_{\|}\right|+\sum_{j}^{\prime} m_{i} v_{i j}\left|\boldsymbol{v}_{j \|}\right|\right] \hat{\boldsymbol{t}}
\end{aligned}
$$

where $-g \sin I=\widehat{t} . g$ and $I$ denotes the magnetic dip angle.

\subsection{The energy conservation equation}

For each ion species the energy conservation equation, considering an isotropic distribution function such that we can replace the stress tensor by a scalar pressure $\left(p_{i}=n_{i} k T_{i}\right)$, can be expressed in terms of the temperature $T_{i}$ as (Bittencourt, 2004)

$$
\begin{aligned}
& \frac{3}{2} n_{i} k\left[\frac{\partial T_{i}}{\partial t}+\left(\boldsymbol{v}_{i} \cdot \nabla\right) T_{i}\right]=-n_{i} k T_{i}\left(\boldsymbol{\nabla} \cdot \boldsymbol{v}_{i}\right)-\nabla \cdot \boldsymbol{q}_{i}+ \\
& M_{i}-m_{i} \boldsymbol{v}_{i} \cdot \boldsymbol{A}_{i}+\left(\frac{1}{2} m_{i} v_{i}^{2}-\frac{3}{2} k T_{i}\right)\left(P_{i}-L_{i}\right)+S_{i}
\end{aligned}
$$

where $\boldsymbol{q}_{i}$ denotes the heat flux vector, $M_{i}$ represents the rate of energy density change due to collisions, $\boldsymbol{A}_{i}$ stands for the collision terms appearing in the equation of motion (7), $P_{i}$ and $L_{i}$ are, respectively, the production and loss terms of the continuity Eq. (1), and $S_{i}$ represents any external energy input (such as solar radiation, for example). In order to use (12), we also need expressions for $\boldsymbol{q}_{i}$ and $M_{i}$ in terms of known quantities.

Equations (1), (7) and (12) constitute a coupled set of nonlinear equations to be solved simultaneously in order to determine the spatial and temporal distribution of the particle number density $n_{i}(r, t)$, macroscopic velocity $\boldsymbol{v}_{i}(r, t)$, and temperature $T_{i}(r, \mathrm{t})$, for each species. In order to simplify matters, instead of solving the energy Eq. (4.3.1), a usual approach consists in considering the temperature distribution for each species, as a function of space and time, as a known quantity. With this approach (considered in the present model), the problem reduces to the solution of the coupled nonlinear set of continuity and momentum conservation equations for each charged particle species. 


\subsection{The divergence terms}

We shall assume that the Earth's magnetic field can be approximated by a centered magnetic dipole, thus having only radial and meridional components. In a region free from electric currents the magnetic induction field can be described in terms of the gradient of a magnetic scalar potential $\gamma$, according to

$\boldsymbol{B}=-\nabla \gamma$

For the geomagnetic dipole approximation we have

$\gamma=g_{0} r_{0}^{3} \frac{\cos \theta}{r^{2}}$

so that,

$\boldsymbol{B}=2 g_{o} r_{o}^{3} \frac{\cos \theta}{r^{3}} \hat{\boldsymbol{r}}+g_{o} r_{o}^{3} \frac{\sin \theta}{r^{3}} \hat{\boldsymbol{\theta}}$

where $\hat{\boldsymbol{r}}$ and $\hat{\boldsymbol{\theta}}$ are unit vectors along the $r$ and $\theta$ directions of a spherical polar coordinate system $(r, \theta, \phi)$ in which $\theta$ denotes the colatitude and the axis $\theta=0^{\circ}$ passes through the north pole. Here $r_{0}$ denotes the Earth's radius and $g_{0}$ is the dipole coefficient, approximately equal to -0.31 gauss.

In addition, the equation of a magnetic field line is

$r=r_{e} \sin ^{2} \theta$

where $r_{e}$ represents the radial distance to the point of intersection of the field line with the geomagnetic equatorial plane. The sense and magnitude of the magnetic dip angle $I$ is taken such that

$\sin (I)=\frac{2 \cos \theta}{\sigma^{1 / 2}}$

$\cos (I)=\frac{\sin \theta}{\sigma^{1 / 2}}$

where $\sigma=\left(1+3 \cos ^{2} \theta\right)$. These expressions can be obtained from Eq. (15), noting that $\tan I=B_{r} / B \theta$. Therefore, we have

$\hat{\boldsymbol{t}}=\sin (I) \hat{\boldsymbol{r}}+\cos (I) \hat{\boldsymbol{\theta}}$

$\nabla_{\|}=\hat{\boldsymbol{t}} \frac{\partial}{\partial s}=\hat{\boldsymbol{t}}\left(\sin (I) \frac{\partial}{\partial r}+\frac{\cos (I)}{r} \frac{\partial}{\partial \theta}\right)$

Kendall (1962) has shown that the basic transport equations for the low-latitude ionosphere are greatly simplified by transforming from the spherical coordinate system $(r, \theta$, $\phi)$ to one whose coordinates define directions parallel and perpendicular to the magnetic field lines. Accordingly, we define a system of orthogonal curvilinear coordinates $(p, q$, $\phi)$ by

$$
\begin{aligned}
& p=\frac{r}{r_{o} \sin ^{2} \theta} \\
& q=\frac{r_{o}^{2} \cos \theta}{r^{2}}
\end{aligned}
$$

Thus, $p=$ constant defines the family of curves which represent the magnetic field lines, while the family of constant magnetic potential surfaces is represented by $q=$ constant. Along a given field line ( $p=$ constant) we have

$$
\begin{gathered}
\frac{\partial}{\partial r}=\frac{\partial q}{\partial r} \frac{\partial}{\partial q} \\
\frac{\partial}{\partial \theta}=\frac{\partial q}{\partial \theta} \frac{\partial}{\partial q}
\end{gathered}
$$

and using Eqs. (21) and (22), together with (17) and (18), the operator $\nabla_{\|}$, given in Eq. (20), becomes

$\nabla_{\|}=\hat{\boldsymbol{t}}(\hat{\boldsymbol{t}} \cdot \nabla)=-\hat{\boldsymbol{t}} \frac{\sigma^{1 / 2} r_{o}^{2}}{r^{3}} \frac{\partial}{\partial q}$

As Kendall (1962) has shown,

$\nabla . \hat{t}=\frac{1}{r \sigma^{3 / 2}}\left(9 \cos \theta+15 \cos ^{2} \theta\right)$

and using the vector identity

$\nabla \cdot\left(n_{i} \boldsymbol{v}_{i \|}\right)=\left(n_{i} v_{i \|}\right) \nabla \cdot \hat{\boldsymbol{t}}+(\hat{\boldsymbol{t}} \cdot \nabla)\left(n_{i} v_{i \|}\right)$

the expression for the divergence of the charged particle flux parallel to the magnetic field lines can be written as

$\nabla .\left(n_{i} \boldsymbol{v}_{i \|}\right)=\left[\frac{1}{r \sigma^{3 / 2}}\left(9 \cos \theta+15 \cos ^{2} \theta\right)-\frac{r_{o}^{2} \sigma^{1 / 2}}{r^{3}} \frac{\partial}{\partial q}\right]\left(n_{i} v_{i \|}\right)$

The expression for $\boldsymbol{v}_{i \|}$, given in Eq. (11), can easily be transformed using (26) and the result incorporated in (28).

\subsection{Coupling to the neutral winds and electric fields}

Since the collisional terms in the charged particle momentum conservation equations involve the thermospheric neutral wind velocity $\boldsymbol{u}$, we must consider the corresponding transport equations for the atmospheric neutral species, which are coupled to the charged particle equations through the collisional terms. The ion drag produced by the neutral wind modifies the ionization distribution, which in turn modifies the neutral wind pattern. Thus, for a self-consistent treatment this coupled system of nonlinear equations must be considered simultaneously. Also, the electric field responsible for the plasma drift in the F-region arises as a result of dynamo action in the E-region, as well as in the F-region.

The E-region dynamo is controlled by the atmospheric tides, and it moves the ionization across the magnetic field lines, since at E-region altitudes the ion collision frequency is much higher than the particle cyclotron frequency. The electric field thus generated maps to the F-region through the high electric conductivity along the field lines.

The thermospheric neutral winds are responsible for the F-region dynamo. However, during the daytime, due to the high conductivity of the E-region, the F-region dynamo is short-circuited through coupling with the E-region via the 
highly conducting magnetic field lines. But, at night, when the E-region conductivity drops drastically, the circuit is open allowing the development of polarization electric fields in the F-region. Again, the electric fields produced modify the ionization distribution in the low-latitude F-region, which in turn modify the pattern of thermospheric neutral winds through ion-neutral drag and, consequently, the electric field. Therefore, a complete self-consistent formulation requires the inclusion of this electrodynamical coupling between the $\mathrm{E}$ and F-regions, responsible for the generation of the Fregion electric fields.

To build a complete self-consistent ionospheric model is an extremely complicated task, requiring the simultaneous numerical solution of a large number of coupled nonlinear differential equations. Furthermore, in the usual approach considered so far, the plasma equations are referred to a Lagrangian geomagnetic coordinate system (in order to simplify the computational procedure), whereas the neutral gas equations are referred to the normal Eulerian geographic coordinate system. Usually, the ionospheric computer models consider the spatial and time variation of both the thermospheric neutral wind velocities and the ionospheric electric fields as known quantities, specified either through analytical formulas or empirical models (e.g. Sterling et al., 1969; Anderson, 1973a; Bittencourt and Tinsley, 1976; Anderson et al., 1987, 1989; Bailey and Sellek, 1990; Batista et al., 1991; Bailey et al., 1993). Also, the early thermospheric neutral wind models considered the spatial and time variation of the ionospheric parameters as known quantities, specified either through analytical formulas or empirical models (e.g. FullerRowell and Rees, 1980). Fuller-Rowell et al. (1987) constructed a coupled thermosphere-ionosphere computer model for high latitudes but, for low latitudes, they still used analytical formulas for the ionospheric variables, given by the empirical ionospheric model of Chiu (1975). Nevertheless, models that treat the fully coupled dynamics and electrodynamics of the ionosphere and thermosphere have been presented by Richmond et al. (1992), Roble and Ridley (1994), Peymirat et al. (1998) and Millward et al. (2001). Also, more general ionosphere models that include mid and high latitudes have been developed. The SAMI2 model calculates the evolution of the low- to mid-latitude ionosphere and was presented by Huba et al. (2000). The Utah State University Time Dependent Ionosphere Model (TDIM) was initially developed as a mid-latitude, multi-ion model by Schunk and Walker (1973), and later as a mathematical model of the middle and high-latitude ionosphere (Schunk, 1988). The theoretical development of the TDIM was described by Schunk (1988), while comparisons with observations were discussed by Sojka (1989).

Regarding the computation of the ionospheric electric fields, some low-latitude computer models have been built. Usually, they solve the equation of motion for the neutral gas together with the equations for the electric fields considering electrodynamical coupling of the equatorial $\mathrm{E}$ and $\mathrm{F}$-regions (e.g. Heelis et al., 1974; Batista et al., 1986). In this case, simplified equations or empirical formulas are used for the ionospheric parameters and conductivities, in order to simplify the computational treatment.

In the low-latitude ionospheric (LION) computer model described here we consider the spatial and time distribution of both the thermospheric neutral wind velocities and neutral densities, and the ionospheric electric fields or $\boldsymbol{E} \times \boldsymbol{B}$ plasma drifts, as known quantities, specified through analytical formulas or empirical models.

\section{Thermospheric neutral winds}

A theoretical description of the global thermospheric neutral wind system requires the numerical solution of a large number of coupled ionospheric and atmospheric equations, involving the time-dependent continuity equations, the momentum conservation equations and the energy conservation equations for each of the ion species as well as for the neutral gas. In addition, to understand also the longitudinal behavior, a three-dimensional solution of this problem is required, taking into account the dependence of the Earth's magnetic field on the geographic coordinates. The forces acting on the neutral air are the pressure gradient force, gravity, frictional forces due to viscosity of the air and due to collisions between the neutral gas particles and the ions (ion-drag), and the Coriolis and centripetal forces due to the Earth's rotation. Since the ion-drag force is proportional to the collision frequency and to the difference between the wind velocity and the ion drift velocity, the various forces that cause ion motion must also be considered simultaneously in a self-consistent way.

\subsection{Basic equations}

The atoms and molecules in the atmosphere collide so frequently that the air can be regarded as a fluid in local thermodynamic equilibrium, described by the usual hydrodynamic conservation equations. Furthermore, the neutral air can be treated as a single fluid, since the macroscopic differential motion of its various constituents is very much less than the overall macroscopic wind velocity. The set of equations governing the dynamics of the neutral upper atmosphere are (Rishbeth, 1972):

(a) The continuity equation for the whole neutral gas, which express the law of mass conservation,

$$
\frac{\partial \rho}{\partial t}+\nabla \cdot(\rho \boldsymbol{u})=0
$$

(b) The Navier-Stokes equation of motion, which express the law of momentum conservation (assuming the air to be incompressible and with constant viscosity),

$$
\frac{\partial \boldsymbol{u}}{\partial t}+(\boldsymbol{u} \cdot \boldsymbol{\nabla}) \boldsymbol{u}+2(\boldsymbol{\Omega} \times \boldsymbol{u})+\boldsymbol{\Omega} \times(\boldsymbol{\Omega} \times \boldsymbol{r})=
$$


$-\frac{1}{\rho} \nabla p-\sum_{i} v_{n i}\left(\boldsymbol{u}-\boldsymbol{v}_{i}\right)+\frac{\mu}{\rho} \nabla^{2} \boldsymbol{u}+\boldsymbol{g}$

(c) The energy conservation equation, neglecting the energy dissipated by viscosity and ion-drag,

$\rho c_{v}\left(\frac{\partial T}{\partial T}+\boldsymbol{u} . \nabla T\right)+p \nabla \cdot \boldsymbol{u}-\nabla \cdot\left(K_{T} \nabla T\right)=P_{E}-L_{E}$

(d) The ideal gas equation of state, which relates pressure, density, and temperature for the neutral air,

$p=n k T$

In this coupled set of equations, $\boldsymbol{u}$ denotes the neutral wind velocity, $\rho$ is the neutral air mass density such that $\rho=n \bar{m}$, $n$ is the neutral air number density, $\bar{m}$ is the average neutral particle mass, $\boldsymbol{v}_{i}$ is the $i$ th ion drift velocity, $\boldsymbol{\Omega}$ is the Earth's angular rotation velocity, $\boldsymbol{r}$ is the radius vector from the center of the Earth to the point where the equations are applied, $p$ stands for the scalar pressure, $v_{n i}$ is the effective neutralion collision frequency, $\mu / \rho$ is the kinematic viscosity coefficient, $\boldsymbol{g}$ represents the acceleration due to gravity, $c_{v}$ is the specific heat at constant volume, $K_{T}$ is the thermal conductivity coefficient, $k$ is Boltzmann's constant and $P_{E}$ and $L_{E}$ represent sources and sinks of energy density. The viscous term in Eq. (30) must be modified if the viscosity coefficient has spatial gradients.

Since the ion-drag term depends on the ion densities (through the collision frequency) and on the ion velocities, the continuity, momentum, and energy equations for each ion species must be considered simultaneously with the neutral gas conservation equations. There has been several numerical model analysis of thermospheric motions. In some of them the temperature field is regarded as a fixed input quantity, usually taken from some phenomenological atmospheric model, such as that of Jacchia $(1965,1971,1977)$. In these cases a treatment of the energy conservation equation is not required. Also, in some cases simplifying approximations are made regarding the ion densities and the ion velocities, which are considered as known fixed quantities, specified as a function of space and local time through a parametric model. Hence, only the set of simplified conservation equations for the neutral air is solved, so that these models are not self-consistent in the sense that the wind pattern modifies the ionization distribution through ion-drag, which in turn modifies the wind pattern. To build a complete and detailed self-consistent model is a formidable task, which requires the simultaneous solution of the three-dimensional system of equations just indicated.

\subsection{Global pressure gradients}

In general the thermospheric neutral winds blow away from the hottest part of the thermosphere, in the afternoon sector, towards the coldest part, in the early morning sector, across the polar regions and zonally around the Earth at low latitudes. This behavior is quite different from that of winds in the lower atmosphere (troposphere), which are controlled by the Coriolis force and the difference is attributed mainly to the importance of ion-drag and viscosity in the upper atmosphere (thermosphere).

To calculate the thermospheric wind velocities from Eq. (30) it is necessary to know the horizontal pressure gradients that provide the driving force for the winds. Clearly, the pressure gradient involves the addictive effects of a density gradient and a temperature gradient, as can be seen from Eq. (32), so that a specified numerical model giving the spatial and time variations of these quantities is required. The global models of the thermosphere usually assume fixed boundary conditions (temperature and neutral species densities) at a lower boundary, often taken as $120 \mathrm{~km}$. The neutral temperature vertical profile is assumed to have a certain shape, tending at great heights to a limiting value, the exospheric temperature $\left(T_{\infty}\right)$, which is a function of local time, latitude, longitude, season, solar activity and magnetic disturbances. The number density vertical profile of each neutral constituent is computed using the diffusive equilibrium equation.

One of the most well known global neutral atmosphere models is that of Jacchia $(1965,1971,1977)$, whose general approach is to determine empirical temperature profiles which yield density distributions in agreement with satellite drag measurements. Jacchia's model provides the global distribution and vertical profiles of the temperature and neutral species densities, with their corresponding time dependences, solar cycle variations, as well as geomagnetic storm atmospheric effects. Other neutral atmosphere models, based on satellite and ground-based observations, are available today, such as the MSIS-86 thermospheric model (Hedin, 1987), as well as models which give the thermospheric neutral wind velocity distribution (e.g. Hedin et al., 1988, 1991) and theoretical global thermospheric models such as that of Fuller-Rowell and Rees (1980).

\subsection{Boundary conditions}

Many atmospheric models used for wind computations assume that the pressure, density and temperature are fixed at some lower boundary level, often taken at $120 \mathrm{~km}$, so that the horizontal pressure gradient vanishes at this boundary. It is therefore generally assumed that the horizontal wind components are equal to zero at this level, viscosity being too weak to vertically transmit any significant horizontal velocity from greater heights. This assumption of unvarying conditions at the lower boundary cannot be expected to be realistic (Chandra and Stubbe, 1970). If the atmospheric parameters $p, \rho$ and $T$ were assumed to vary at the lower boundary, as they probably do in reality, some effect would be observed in the computed winds at greater heights.

Since the kinematic viscosity $(\mu / \rho)$ becomes very large at great heights, the derivative $\left(\partial^{2} \boldsymbol{u} / \partial z^{2}\right)$ must become small at these heights, in order that the viscosity term in the equation 
of motion (30) should not become overwhelmingly large. This implies that we must have $(\partial \boldsymbol{u} / \partial z) \rightarrow$ constant. Further, to maintain a finite velocity gradient there would have to exist a shearing force which neither the pressure gradients nor the Coriolis force nor ion-drag can provide, so that in fact, $\partial \boldsymbol{u} / \partial z=0$ at great heights, i.e. $\boldsymbol{u}$ becomes heightindependent, which is the upper boundary condition for the neutral air equation of motion.

\subsection{Thermospheric neutral wind models}

As mentioned before, in the low-latitude ionospheric computer model described here, the horizontal thermospheric neutral wind velocity field is considered to be specified through some analytical or empirical expression, or even given through a numerical model, such as the MSIS-86 thermospheric model (Hedin, 1987). As the atmosphere undergoes thermal contraction and expansion, the vertical velocity of a surface of constant pressure is given by (Jacchia, 1977)

$u_{r}=\frac{\Omega T}{g} \int_{z_{0}}^{z} \frac{g}{T^{2}} \frac{\partial T}{\partial \phi} d z^{\prime}$

Taking the temperature $\mathrm{T}$ as being the exospheric temperature $T_{\infty}$, independent of altitude, we have

$u_{r}=\frac{\Omega}{T_{\infty}} \frac{\partial T_{\infty}}{\partial \phi} \frac{\left(z-z_{0}\right) r}{\left(R_{E}+z_{0}\right)}$

The neutral air wind velocity along $\boldsymbol{B}$ can be expressed as

$\boldsymbol{u}_{\|}=\left(u_{r} \sin I+u_{\theta} \cos I\right) \hat{\boldsymbol{t}}$

Here $u_{r}$ is the radial velocity of the neutral air due to the diurnal expansion and contraction of the atmosphere, given by expression (33), and $u_{\theta}$ is the magnetic north-south component of the horizontal thermospheric neutral wind velocity, relative to the Earth.

The horizontal wind velocity component along the magnetic meridian $\left(u_{\theta}\right)$ can be expressed in terms of the geographic components of the horizontal thermospheric wind velocity as

$u_{\theta}=u_{\theta}^{\prime} \cos \delta_{m}+u_{\phi}^{\prime} \sin \delta_{m}$

where $u_{\theta}^{\prime}$ represents the geographic meridional wind component, $u_{\Phi}^{\prime}$ represents the geographical zonal wind component and $\delta_{m}$ stands for magnetic declination, which is greatly longitudinal dependent.

Notice that the ionospheric plasma equations are solved along a given magnetic field line, so that the proper wind component to be used in the plasma equations is the magnetic meridian component $u_{\theta}$, with its longitudinal dependence on magnetic declination already included according to (35).

A number of thermospheric neutral wind models, giving the space and time dependence of $u_{\theta}^{\prime}$ and $u_{\Phi}^{\prime}$, can be considered for the present ionospheric model calculations. A recent one is the global thermospheric wind model of Hedin et al. (1991, 1988), based on satellite and ground-based observations. A very simple analytical expression for the thermospheric wind velocity field, represented by an asymmetrical cosine function in the local time dependence, with an amplitude which increases with latitude (Bittencourt, 1996), may also be considered,

$u_{\theta}=u_{0}\left[\frac{1-\sin (\theta-\Delta)}{1-\sin \theta_{0}}\right]\left[\cos \left(\phi+\phi_{0}\right)+\epsilon\right]$

In this expression, $u_{0}$ is a constant velocity, $\theta$ is the magnetic colatitude, $\theta_{0}$ is a normalization constant, the parameter $\Phi_{0}$ determines the local time at which the wind achieves its maximum velocity, $\varepsilon$ permits a choice of smaller velocities during the daytime as compared to the nighttime, allowing for the effect of great ion drag during the day, and $\Delta$ represents the latitudinal difference between the position of the magnetic equator at a particular longitude and the latitude to which the winds converge or diverge in their global pattern. Thus, for equinox conditions, $\Delta$ represents the geographic latitude of the location of the magnetic equator at a fixed longitude. Further, up to $14^{\circ}$ can be considered for the separation between the magnetic and geographic equators, depending on the longitude chosen, and another $23^{\circ}$ for the movement of the sub-solar point, depending on the season. An expression similar to Eq. (36) was previously used by Sterling et al. (1969), and by Brasher and Hanson (1970) on previous tropical F-region models, but only for the simple case of equinox and coincidence of geomagnetic and geographic equators.

Different wind representations (considered as input parameters specified as a function of space and time) can be used in the LION model in order to analyze the wind effects on the low-latitude ionospheric ionization distribution at the various longitudes, and for different seasons and solar activity.

\section{Electromagnetic plasma drifts}

In the ionospheric E-region the motions of the neutral air, caused by atmospheric tides, are able to transport (through collisions) the ionization across the magnetic field lines causing currents to flow. Polarization fields (electrostatic) are generated which affect the motion of the charged particles in the ionospheric F-region and in the magnetosphere. Since the electrical conductivity along the magnetic field lines is very high, they can be thought of as equipotential wires which transmit electric fields from one region to another (Farley, 1959).

Rishbeth (1971) suggested that thermospheric neutral winds may generate F-region currents (see also Rishbeth 1977, 1981). The resultant polarization fields may or may not be shorted out in the E-region. During the day the Eregion ionization is sufficient to short circuit these polarization fields, but at night the very low E-region electron densities allow the field to develop. Consequently, a vertical 
electric field is established in the equatorial F-region, by the zonal thermospheric winds, causing the ionization to drift in the east-west direction. This plasma drift is in the same direction as the neutral wind that produces the polarization field.

The electrostatic component $\boldsymbol{E}$ of the total electric field $(\boldsymbol{E}+\boldsymbol{u} \times \boldsymbol{B})$ gives rise to the drifts of the F-region plasma. At the magnetic equator, the east-west component of $\boldsymbol{E}$ generates the vertical $\boldsymbol{E} \times \boldsymbol{B}$ ionization drift, which is upwards during the daytime and downwards at night. The north-south component of the E-region electric field when transmitted to the F-region, over the magnetic equator, points in the vertical direction, giving rise to an east-west $\boldsymbol{E} \times \boldsymbol{B}$ plasma drift. Review articles on the equatorial ionospheric electric fields and low-latitude electrodynamic plasma drifts were published by Fejer (1981, 1991, 1997).

\subsection{Divergence of the plasma drift velocity}

In the F-region, the electric field $\boldsymbol{E}$ that exists normal to the magnetic field, as a result of dynamo action in the E-region, produces a drift velocity of the plasma across the magnetic field lines given by

$\boldsymbol{v}_{\perp}=\frac{\boldsymbol{E} \times \boldsymbol{B}}{B^{2}}$

The electric field responsible for this drift can be separated into two parts

$\boldsymbol{E}=\boldsymbol{E}_{\mathrm{cor}}+\boldsymbol{E}_{d}$

where $\boldsymbol{E}_{\text {cor }}$ is such that the velocity $\left(\boldsymbol{E}_{\text {cor }} \times \boldsymbol{B}\right) / B^{2}$ gives corotation with the Earth, i.e.,

$$
\frac{\boldsymbol{E}_{\text {cor }} \times \boldsymbol{B}}{B^{2}}=r \sin \theta \Omega \hat{\boldsymbol{\phi}}
$$

where $\Omega$ denotes the Earth's angular velocity, $\hat{\phi}$ represents a unit vector in the $\phi$-direction and $\boldsymbol{E}_{d}$ is the electric field normally associated with the ionospheric dynamo system.

The plasma drift velocity can be resolved into the form

$\boldsymbol{v}_{\perp}=v_{n} \hat{\boldsymbol{n}}+\left(v_{\phi}+r \sin \theta \Omega\right) \hat{\boldsymbol{\phi}}$

where $\hat{\boldsymbol{n}}=\cos I \hat{\boldsymbol{r}}-\sin I \hat{\boldsymbol{\theta}}$, which represents a unit vector in the vertical plane, normal to the magnetic field line, and $v_{n}$ and $v \phi$ are the components of $\boldsymbol{v}_{\perp}$ in the vertical plane and in the east-west direction, respectively, relative to the Earth. Therefore,

$$
\begin{aligned}
& v_{n} \hat{\boldsymbol{n}}=\frac{1}{B^{2}}\left[\left(\boldsymbol{E}_{d} \times \boldsymbol{B}\right) \cdot \hat{\boldsymbol{r}}\right] \hat{\boldsymbol{r}}+\frac{1}{B^{2}}\left[\left(\boldsymbol{E}_{d} \times \boldsymbol{B}\right) \cdot \hat{\boldsymbol{\theta}}\right] \hat{\boldsymbol{\theta}} \\
& v_{\phi} \hat{\boldsymbol{\phi}}=\frac{1}{B^{2}}\left[\left(\boldsymbol{E}_{d} \times \boldsymbol{B}\right) \cdot \hat{\boldsymbol{\phi}}\right] \hat{\boldsymbol{\phi}}
\end{aligned}
$$

and the divergence of the $\phi$-component is

$\nabla \cdot\left(r \sin \theta \Omega \hat{\boldsymbol{\phi}}+v_{\phi} \hat{\boldsymbol{\phi}}\right)=\frac{1}{r \sin \theta} \frac{\partial v_{\phi}}{\partial \phi}$
Sterling et al. (1969) showed that the effect of $v_{\phi}$ in the solutions is negligible so that in the numerical computer calculations it can be assumed that $v_{\phi}=0$. Therefore, only drifts in the magnetic meridional plane, due to an east-west electric field, are considered in the LION model, even though eastwest plasma drifts are known to exist.

The divergence of the component of $\boldsymbol{v}_{\perp}$ in the vertical plane can be expressed as (Baxter, 1964; Moffett and Hanson, 1965)

$$
\nabla \cdot\left(v_{n} \hat{\boldsymbol{n}}\right)=\frac{\partial v_{n}^{0}}{\partial r_{e}}+\frac{4 v_{n}^{0}}{r \sigma^{2}}\left(6 \cos ^{6} \theta-3 \cos ^{4} \theta-4 \cos ^{2} \theta+1\right)
$$

where $v_{n}^{0}$ is the equatorial value of $v_{n}, r_{e}$ is the radial distance from the center of the Earth to the field line's equatorial crossing point and $\sigma=\left(1+3 \cos ^{2} \theta\right)$.

The radial dependence of the vertical drift at the dipole equator is given by (Sterling et al., 1972)

$v_{n}^{0}=v_{0} \frac{r^{2}}{\left(h_{0}+r_{0}\right)^{2}}$

where $r_{0}$ denotes the Earth's radius and $v_{0}$ is the plasma drift velocity at $h_{0}=300 \mathrm{~km}$ above the surface at the dip equator, i.e., the drift velocity normally measured at Jicamarca with the incoherent scatter radar, for example. This radial squared dependence in $v_{n}^{0}$ is chosen so that the magnetic flux in the field tube is conserved as the plasma moves vertically.

\subsection{Plasma drift velocity measurements}

Measurements of vertical plasma drifts at the magnetic equator have been reported by Woodman (1970) and Fejer et al. $(1989,1991)$, obtained using the incoherent scatter radar at Jicamarca, Peru. Typically, upward velocities of $20 \mathrm{~m} / \mathrm{s}$ to $25 \mathrm{~m} / \mathrm{s}$ are observed during the day and downward velocities of about the same magnitude at night. A rapid increase in the upward velocity, commencing around sunset and lasting between one to two hours, is also observed which is a consistent feature appearing every day with regularity, after which the velocities reverse to downward. Typical velocities at this pre-reversal peak may be as high as $40 \mathrm{~m} / \mathrm{s}$. The amplitude and duration of this pre-reversal peak in the upward velocities vary from one longitudinal region to another and with season, showing a marked dependence on magnetic declination. Woodman (1970) found that the spread in the velocities at any one time, for different days, is as large as the velocities themselves, even during magnetically quiet days, and that the daily behavior of the drift velocities is far from sinusoidal. Also, Fejer et al. (1979) investigated the effects of geomagnetic disturbances on the vertical electromagnetic plasma drifts, finding that in general, in most cases during geomagnetic storm conditions, the drifts are somewhat inhibited. Woodman (1972) has measured the east-west electromagnetic plasma drift component, finding that the plasma drifts westward during the day with a typical 
velocity of about $50 \mathrm{~m} / \mathrm{s}$ and eastward at night with velocities from $100 \mathrm{~m} / \mathrm{s}$ to $150 \mathrm{~m} / \mathrm{s}$.

Bittencourt and Abdu (1981) found that when the Flayer is sufficiently high, such that transport processes dominate over recombination (above about $300 \mathrm{~km}$ ), the vertical plasma drifts can be determined to good accuracy from the vertical motions of the F2-peak height, as determined from ionosonde measurements. This technique allows the determination of the vertical plasma drifts in different longitudinal regions to investigate their magnetic declination and seasonal dependence, at least in the hours near sunset and early evening when the F-layer is sufficiently high. This method, however, underestimates the vertical plasma drifts when the F-layer is not high enough, due to the effects of plasma recombination (Bittencourt and Abdu, 1981; Fejer et al., 1989; Batista et al., 1990).

\subsection{Theoretical models}

Some theoretical models involving the calculation of electric fields, and the corresponding electric potentials, in the low-latitude ionosphere have been developed (e.g. Heellis et al., 1974; Batista et al., 1986). These models consider that the equatorial F-region electric field is generated by the atmospheric tides, through the E-region dynamo, and by the thermospheric winds, through the F-region dynamo and the electrodynamical coupling between the $\mathrm{E}$ and F-regions. The basic equations include the conservation equations for the neutral air and for the ionospheric ionization, Maxwell equations and the equation for the electric current flow that provides the electrodynamical coupling between the $\mathrm{E}$ and F-regions. The models developed by Heellis et al. (1974) and by Batista et al. (1986) assume various simplifying approximations for this set of equations in order to reduce the complexity of its numerical solution. A global vertical drift model for the equatorial F-region was recently published by Scherliess and Fejer (1999). Fejer et al. (2005) presented an empirical model of equatorial zonal plasma drifts near the F-region, over Jicamarca.

In the LION model we consider the F-region electromagnetic plasma drifts as known quantities and specified as a function of space and time through analytical or empirical formulas, based on incoherent scatter and ionosonde observations, as well as on numerical modeling. Different drift models can be considered in order to provide an adequate representation for the drift dependence on longitude (magnetic declination) and season, as well as on solar activity.

\section{Photoionization and ion chemistry}

\subsection{Photoionization rates}

The photoionization rate per unit volume for each of the absorbing atmospheric species, produced by the solar ionizing radiation, can be expressed as

$Q_{j}(r, \chi)=\sum_{k} \Phi_{\infty}\left(\lambda_{k}\right) \exp \left[-\tau\left(\lambda_{k}, r, \chi\right)\right] \sigma_{j}^{(i)}\left(\lambda_{k}\right) n_{j}(r)(47)$

where $\Phi_{\infty}\left(\lambda_{k}\right)$ represents the incident solar extreme ultraviolet (XUV) radiation flux, in the wavelength band specified by $\lambda_{k}$, at the top of the atmosphere where the optical depth $\tau(\lambda \mathrm{k}, \mathrm{r}, \chi)$ is zero, $\sigma_{j}^{(i)}$ denotes the photoionization cross section in the wavelength band $\lambda_{k}$ for the $j$ th absorbing atmospheric species, $\mathrm{r}$ is the radial distance, $n_{j}(r)$ is the number density of the $\mathrm{j}^{\text {th }}$ species, $\chi$ is the solar zenith angle and the summation applies over all wavelength bands of incident solar XUV radiation. The total photoionization rate per unit volume is obtained by summing Eq. (7.1.1) over all absorbing species,

$Q_{T}(r, \chi)=\sum_{j} Q_{j}(r, \chi)$

The exponential part in (46) represents the attenuation of the solar radiation produced by the atmosphere above the altitude considered. The optical depth can be expressed as

$\tau\left(\lambda_{k}, r, \chi\right)=\sum_{j} \int_{r}^{\infty} \sigma_{j}^{(a)}\left(\lambda_{k}\right) n_{j}\left(r^{\prime}\right) C h\left(r^{\prime} / H_{j}, \chi\right) d r^{\prime}$

where $H_{j}$ is the scale height of the $j$ th constituent, $C h\left(r^{\prime} / H_{j}, \chi\right)$ denotes the geometrical Chapman function which takes into account the Earth's sphericity and $\sigma_{j}^{(a)}$ $\left(\lambda_{k}\right)$ stands for the absorption cross section in the wavelength band $\lambda_{k}$ for the $\mathrm{j}^{\text {th }}$ absorbing species. Equation (48) can be replaced by the following approximated simplified expression

$\tau\left(\lambda_{k}, r, \chi\right)=\sum_{j} \sigma_{j}^{(a)}\left(\lambda_{k}\right) n_{j}(r) H_{j} C h\left(r / H_{j}, \chi\right)$

For each of the absorbing species, taken to be $\mathrm{O}, \mathrm{O}_{2}$ and $\mathrm{N}_{2}$, the photoionization rate per unit volume is computed from expression (46), considering 62 discrete wavelength intervals in the range from $3.0 \mathrm{~nm}$ to $102.6 \mathrm{~nm}$.

The incident solar XUV radiation flux $\Phi_{\infty}\left(\lambda_{k}\right)$ and the absorption and ionization cross sections, $\sigma_{j}^{(a)}\left(\lambda_{k}\right)$ and $\sigma_{j}^{(i)}$ $\left(\lambda_{k}\right)$, used in previous models were taken from the results published by Hinteregger et al. (1965), which are based on satellite and rocket observations. Hinteregger (1970) has suggested that these solar XUV radiation fluxes, measured under solar minimum conditions (in 1963), are probably more representative of solar maximum conditions. Solar EUV flux models have also been published by Tobiska and Barth (1990) and by Tobiska (1991). The solar fluxes and ionization cross sections published by Tobiska (1991) have been used to generate the results presented here.

The optical depth for each wavelength band is calculated from expression (49), considering the summation (index $j$ ) 
over the atmospheric species $\mathrm{O}, \mathrm{O}_{2}$ and $\mathrm{N}_{2}$. Due to the predominance of atomic oxygen above about $250 \mathrm{~km}$, it constitutes the dominant term in the calculation of the optical depth.

\subsection{Ion chemistry}

Loss of ionization in the ionospheric F-region is controlled by recombination processes such as electron-ion and ion-ion recombinations. The pertinent reactions in these loss mechanisms include radiative and dissociative recombinations. Ionatom interchange and charge exchange reactions are also efficient and must be considered in both production and loss rates for the ion species. Ion-atom interchange reactions are generally more rapid than charge exchange reactions (Bates, 1955).

The ionization production and loss rates per unit volume, resulting from ion chemistry, are governed by the rate coefficients of the relevant ion-ion, ion-neutral and ion-electron processes. The ionic reactions considered in the LION model and the magnitude of their reaction rate coefficients are presented in Bittencourt (1996).

\section{Neutral atmosphere model}

Several model atmospheres, based on experimental data from satellites and from the basic equations governing atmospheric structure, have been developed, which provide the spatial and time dependence of the neutral gas temperature and neutral species concentrations in the upper atmosphere, including seasonal, solar cycle and geomagnetic activity dependences (e.g. Jacchia, 1965, 1971, 1977; Hedin, 1987).

A simple analytical model for the neutral atmosphere is that based on the Jacchia (1977) atmospheric model, combined with Walker's (1965) analytic expressions for the temperature and density profiles. The modification incorporated by Walker (1965) avoids the numerical integration of the diffusive equilibrium equations for each neutral species. Another possible approach is to use the MSIS-86 thermospheric model of Hedin (1987), which is based on satellite data.

\subsection{Temperature profiles}

Jacchia's expression for the global distribution of exospheric temperature, $T_{\infty}$, is

$T_{\infty}=T_{0}\left(1+R \sin ^{m} \psi\right) \cdot\left[1+R \frac{\left(\cos ^{m} \eta-\sin ^{m} \psi\right)}{\left(1+R \sin ^{m} \psi\right)} \cos ^{n}\left(\frac{\tau}{2}\right)\right]$

where $T_{0}$ is the minimum nighttime exospheric temperature, $(1+R) T_{0}$ is the maximum daytime value of the exospheric temperature, $\mathrm{m}$ and $\mathrm{n}$ are constants, $\eta$ and $\psi$ are functions of geographic latitude $(\Lambda)$ and solar declination $\left(\delta_{0}\right)$, defined by

$\eta=\left(\Lambda-\delta_{0}\right) / 2$
$\psi=\left(\Lambda-\delta_{0}\right) / 2$

and the parameter $\tau$ is a function of local time defined according to

$\tau=H+\beta+p \sin (H+\gamma) ;(-\pi<\tau<\pi)$

where $H$ represents the solar hour angle measured from noon, in radians, and $\beta, p$ and $\lambda$ are constants which specify the phase of maximum exospheric temperature and the shape of the isotherms of exospheric temperature over the globe. The quantity $T_{0}$ is dependent on solar activity.

The temperature profile can be calculated, according to Walker (1965), from

$T=T_{\infty}-\left(T_{\infty}-T_{120}\right) \exp (-\sigma \xi)$

where $T_{120}$ is the temperature at $120 \mathrm{~km}$ and $\sigma$ is an analytical function of $T_{\infty}$ given (in $\mathrm{km}^{-1}$ ) by

$\sigma=0.0291 \exp \left(-X^{2} / 2\right)+\left(r_{0}+120\right)^{-1}$

where $r_{0}$ is the Earth's radius (in $\mathrm{km}$ ) and

$X=\frac{\left(T_{\infty}-800\right)}{750+1.722 \times 10^{-4}\left(T_{\infty}-800\right)^{2}}$

The geopotential altitude, $\xi$, is given (in $\mathrm{km}$ ) by

$\xi=\frac{(z-120)\left(r_{0}+120\right)}{\left(r_{0}+z\right)}$

in which $z$ represents the altitude, above the Earth's surface, of the point considered.

\subsection{Neutral density profiles}

The diffusive equilibrium equation can be integrated analytically using the temperature profile given in (54). Walker (1965) obtained the following expression for the number density of the $\alpha$ neutral species

$n_{\alpha}(z)=n_{\alpha}(120)\left[\frac{(1-a)}{1-a \exp (-\sigma \xi)}\right]^{(1+\gamma)} \exp \left(-b_{\alpha} \sigma \xi\right)$

where

$a=\frac{\left(T_{\infty}-T_{120}\right)}{T_{\infty}}$

$b_{\alpha}=\frac{m_{\alpha} g_{120}}{\sigma k T_{\infty}}$

$k$ is Boltzmann's constant, $g_{120}$ stands for the gravitational acceleration at the $120 \mathrm{~km}$ base level and $m_{\alpha}$ is the mass of the neutral constituent $\alpha$.

Values for the neutral atmospheric parameters used in the LION model can be found in Bittencourt (1996). 


\section{Diffusion rates and collision frequencies}

The general theory for diffusion of ions through a gas was originally developed by Chapman (1939). In the low-latitude ionosphere the relevant ions $\mathrm{O}^{+}, \mathrm{O}_{2}^{+}, \mathrm{NO}^{+}, \mathrm{N}_{2}^{+}$and $\mathrm{N}^{+}$diffuse through the gases of the neutral atmosphere and through each other. The force per unit volume acting on the $\mathrm{i}^{\text {th }}$ species, due to collisions, is given by

$\boldsymbol{f}_{\mathrm{coll}}^{(i)}=-\sum_{j}^{\prime} m_{i} n_{i} v_{i j}\left(\boldsymbol{v}_{i}-\boldsymbol{v}_{j}\right)-\sum_{n} m_{i} n_{i} v_{i n}\left(\boldsymbol{v}_{i}-\boldsymbol{u}\right)(62)$

where the first summation is over all ion species, except the $i$ th, and the second one is over all neutral atmospheric species.

The collision frequencies used in the present computer model are derived from the relationship

$v_{i j}=\frac{k T_{i} n_{j}}{m_{i} b_{i j}}$

where the $b_{i j}$ 's are the binary collision parameters. The values for the binary collision parameters used in the LION model are the ones given in Bittencourt (1996) and have been derived from the individual ion mobilities in a neutral gas as given by Dalgarno $(1961,1964)$. The temperature dependence of the ion-ion collision parameters was considered to be $\left(T_{i} / 1500\right)^{5 / 2}$ and the dependence of the ion-neutral collision parameters was $\left(T_{n} / 300\right)^{1 / 2}$, with the temperatures expressed in degrees Kelvin. Furthermore, in all calculations we take $T_{i}=T_{e}=T_{n}$.

The ion diffusion coefficient $D_{i}$ and the collision frequency $v_{i j}$ are related through the expression

$D_{i}=\frac{k T_{i}}{m_{i}\left(\sum_{j} v_{i j}\right)}=\left[\sum_{j}\left(n_{j} / b_{i j}\right)\right]^{-1}$

The summation in Eq. (63) applies to both ions and neutrals.

\section{Computational procedure}

\subsection{Variable transformations}

In order to simplify the equations and to put them in a form suitable for numerical solution, three variable transformations are made. These variable transformations are dictated mainly by numerical stability considerations, speed of computation and convenience in interpreting the results.

The first transformation involves the change of the independent variable time t, to longitude $\phi$, which allows a straightforward interpretation of the results at specified local times, according to

$\frac{\partial n_{i}}{\partial \phi}=\frac{1}{\left(v_{\phi} / r+\Omega\right)} \frac{\partial n_{i}}{\partial t}$
The second transformation maps the parameter $q$ into a parameter $Y$, defined by

$Y=\frac{\sinh (\Gamma q)}{\sinh \left(\Gamma q_{\max }\right)}$

where $\Gamma$ is a suitably chosen number and $q_{\max }$ is the value of $q$ at the northern end of the field line, where $r=r_{b}$, and $r_{b}$ is some base value of $r$. This base level in the present model is taken at $120 \mathrm{~km}$ and $\Gamma=10$. Equal increments in $q$ give too many points at high altitudes and not enough near the F2-peak. This transformation maps the magnetic field lines into straight lines with $Y=1$ at the northern end (where $\left.q=q_{\max }\right), Y=0$ at the dipole equator and $Y=-1$ at the southern end (where $q=-q_{\max }$ ).

The third transformation replaces the dependent variable $n_{i}(r, t)$ by the variable $G_{i}(r, t)$, defined by

$G_{i}=n_{i} \exp \left(\int_{r_{0}}^{r} \frac{d r^{\prime}}{\alpha H_{i}}\right)$

where $\alpha=\left(T_{e}+T_{i}\right) / T_{i}$ and $H_{i}$ is the ion scale height. This transformation improves the stability of the numerical solutions since, at great altitudes where $n_{i}(r, t)$ varies in an exponential manner, $G_{i}(r, t)$ is essentially constant along a magnetic field line. It can be applied to any of the ions considered here, but its use was restricted to the $\mathrm{O}^{+}$ions only, which is the dominant ion above the F2-peak in the low-latitude ionospheric F-region.

These transformations are incorporated in the equations according to the details given in Bittencourt (1996). The resultant system of coupled partial differential equations is solved using an iterative, implicit finite-difference method, similar to method three of Crank and Nicolson (1947) (see also Potter, 1980).

\subsection{Boundary conditions, spatial grid, and time step}

At all times the boundary conditions at $y= \pm 1$ (base level) are $n_{i}(\phi, y)=0$, while at $t=0$ some initial ionization distribution is assumed everywhere along the field line. After a few integration steps in time, the solution becomes independent of the initial values adopted, because of the effects of photoionization, ion chemistry and plasma transport.

After the transformation of the coupled set of non-linear differential equations into a discrete numerical set of finitedifference equations, we must specify the time step and spatial grid to be used in the numerical computations. A usual step in $\phi$ (local time) is $1^{\circ}$ (corresponding to $4 \mathrm{~min}$ ), but in some cases a smaller step may be considered, depending on the ionospheric phenomena under analysis. Along the magnetic field line 99 steps are used in $y$ (50 in each hemisphere, with one common point at the magnetic equator). It is appropriate to start the time integration around 08:00 local time, cover a full 24-h period, ending about two or three hours past 08:00 local time of the next day, when the calculation results start to repeat themselves for the same local time. The 
calculation results for the first two or three hours are then neglected in order to eliminate any possible influence of the initial values adopted. In this sense, the results (densities and velocities for ions and electrons) obtained for a complete day $(24 \mathrm{~h})$, along a given field line (moving vertically at the electromagnetic plasma drift velocity in the Lagrangian frame of reference), must repeat themselves for the next $24 \mathrm{~h}$, when the computer program is asked to run in sequence, so that there is a $24-\mathrm{h}$ periodicity in the results.

In order to be able to construct vertical profiles of the particle number densities and velocities, over a latitudinal range between about $\pm 20^{\circ}$, the integration in $\phi$ is repeated over the 24-h period for a given number of magnetic field lines (about one hundred or more field lines) with their equatorial crossing altitude chosen in such a way as to cover the altitude range of interest, for all times, in the latitudinal range considered, as illustrated in Fig. 1. The distribution in height of the starting magnetic field lines (equatorial crossing altitude of each field line) is selected such that the vertical ionization distribution in the region of the equatorial Appleton anomaly (between at least $\pm 15^{\circ}$ north-south) can be accurately constructed for all $\phi$-steps.

For each step in $\phi$ (local time) a two-dimensional interpolation scheme is employed to transform the particle number densities and velocities along the magnetic field lines into a uniform grid in height and magnetic latitude. A twodimensional grid is then constructed at $5 \mathrm{~km}$ (or less) increments in height and $0.5^{\circ}$ increments in magnetic latitude using a three-point Lagrange interpolation scheme. This interpolation is carried out first in height along each field line and then in latitude for each height level using the various field lines. These results are then graphically processed with appropriate softwares in order to generate different types of color graphic representations for adequate visualization of the dynamics of the various ionospheric phenomena of interest.

\section{Model results}

In this section we shall give some idea of the typical results generated by the LION model, in order to illustrate its potential applicability in the study of a variety of important ionospheric phenomena at low latitudes. The model runs in a normal personal computer (PC) and outputs the results in numerical format as well as in graphic form for each instant of time, thus allowing a continuous visualization of the time evolution of the ionospheric ionization distribution in space.

For illustration purposes, Fig. 2 shows the spatial variation of the F2-region electron density in a height and magnetic latitude grid, for various local times, generated by the LION model for average solar conditions in the eastern Brazilian longitudinal region, during summer in the Southern Hemisphere. In the results shown here we have used the electromagnetic vertical plasma drift velocities published by Batista

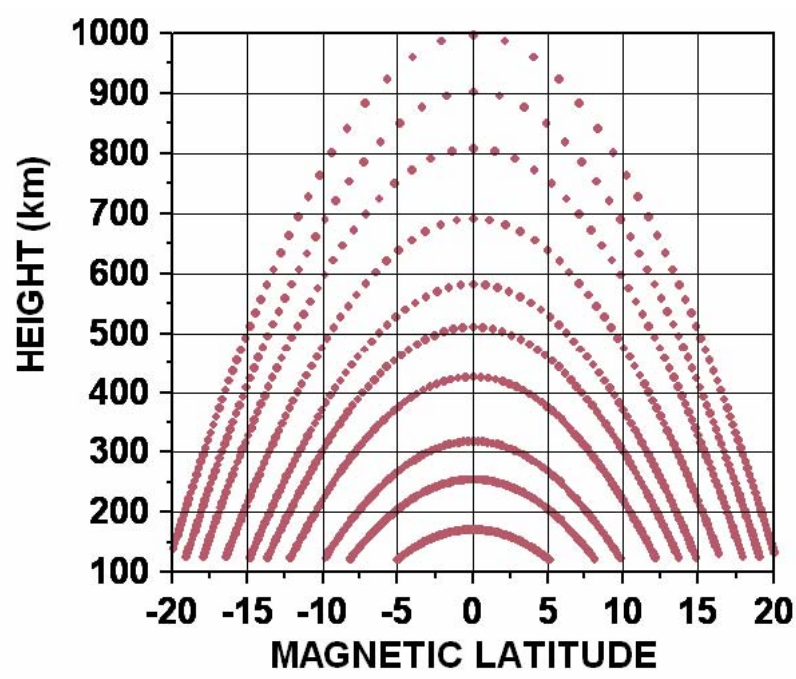

Fig. 1. Set of dipole magnetic field lines illustrating the height versus magnetic latitude grid used in the LION model, with 99 points along each field line.

et al. (1996), which are based on Jicamarca incoherent scatter measurements (Woodman, 1972, 1970), and ionosonde observations over the Brazilian sector for the pre-reversal peak velocities. For the neutral wind model we used the analytical expression given in Eq. (36), taking into account the separation between the geomagnetic and geographic equators and the magnetic declination for the longitudinal region considered (eastern Brazilian sector). For more details on the wind and drift models, refer to Bittencourt (1996). It must be stressed, however, that the LION model allows the inclusion of external empirical wind models based on satellite and ground-based observations, that give the thermospheric neutral wind velocity distribution (e.g. Hedin et al., 1988, 1991) and theoretical global thermospheric models such as that of Fuller-Rowell and Rees (1980). Figure 3 shows similar results, but for equinox conditions. Similar maps can also be generated for all the ions considered in the model, as well as maps showing the density and velocity variations in different space and local time grids. The F2-peak ionospheric electron density $\left(n_{m}\right)$ as a function of magnetic latitude and local time, generated by the LION model for average solar conditions in the Brazilian longitudinal region, is shown in Fig. 4 (for summer conditions in the Southern Hemisphere), and in Fig. 5 (for equinox conditions).

The electromagnetic vertical plasma drift at low latitudes is the main process responsible for the formation of the Appleton ionospheric equatorial anomaly, as discussed earlier. In general terms, as the vertical upward drift increases, the latitudinal separation of the anomaly north-south crests increases, at the same time that the crest-to-trough ratio in the electron density also increases. The enhancement in the vertical plasma drift that occurs just after sunset (known as 

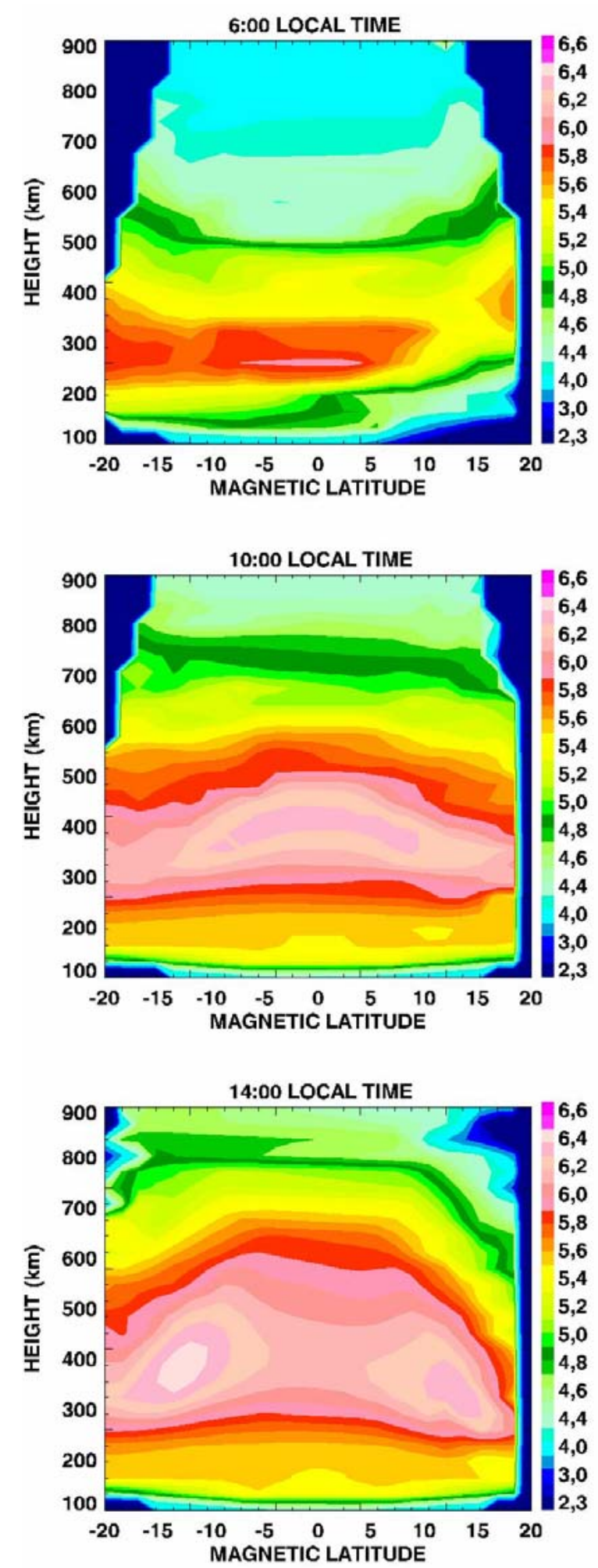
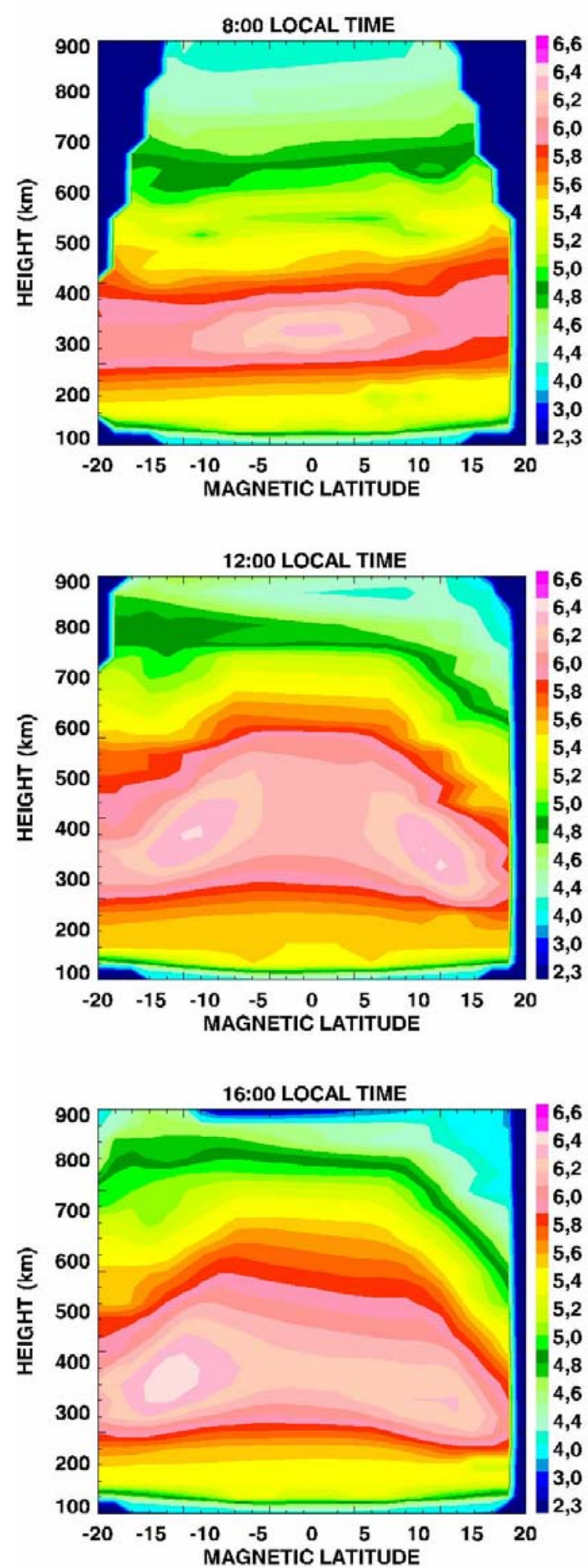

Fig. 2. Ionospheric electron density distribution as a function of height and magnetic latitude for various local times generated by the LION model for average solar conditions in the Brazilian longitudinal region, during summer in the Southern Hemisphere. 

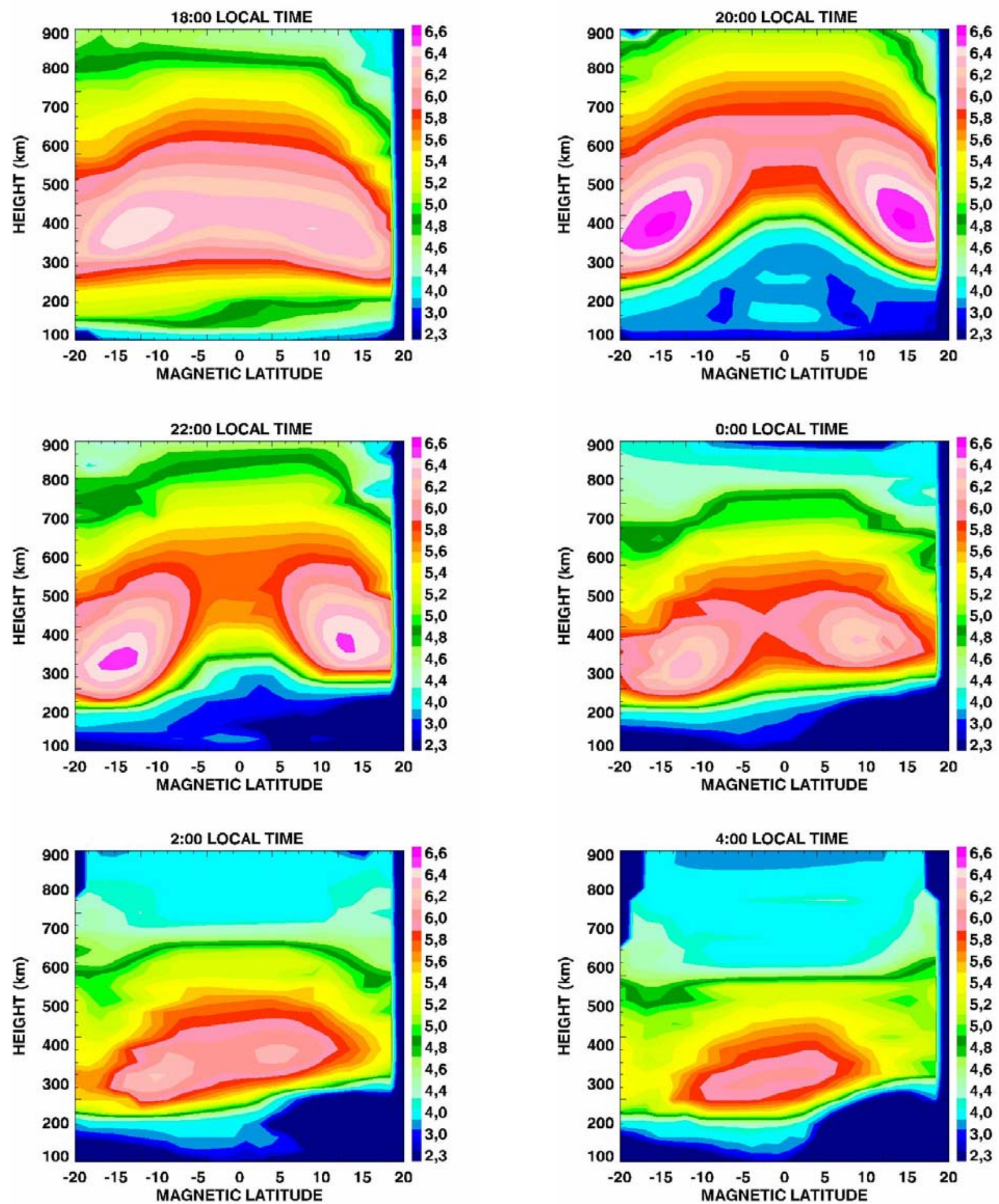

Fig. 2. Continued. 

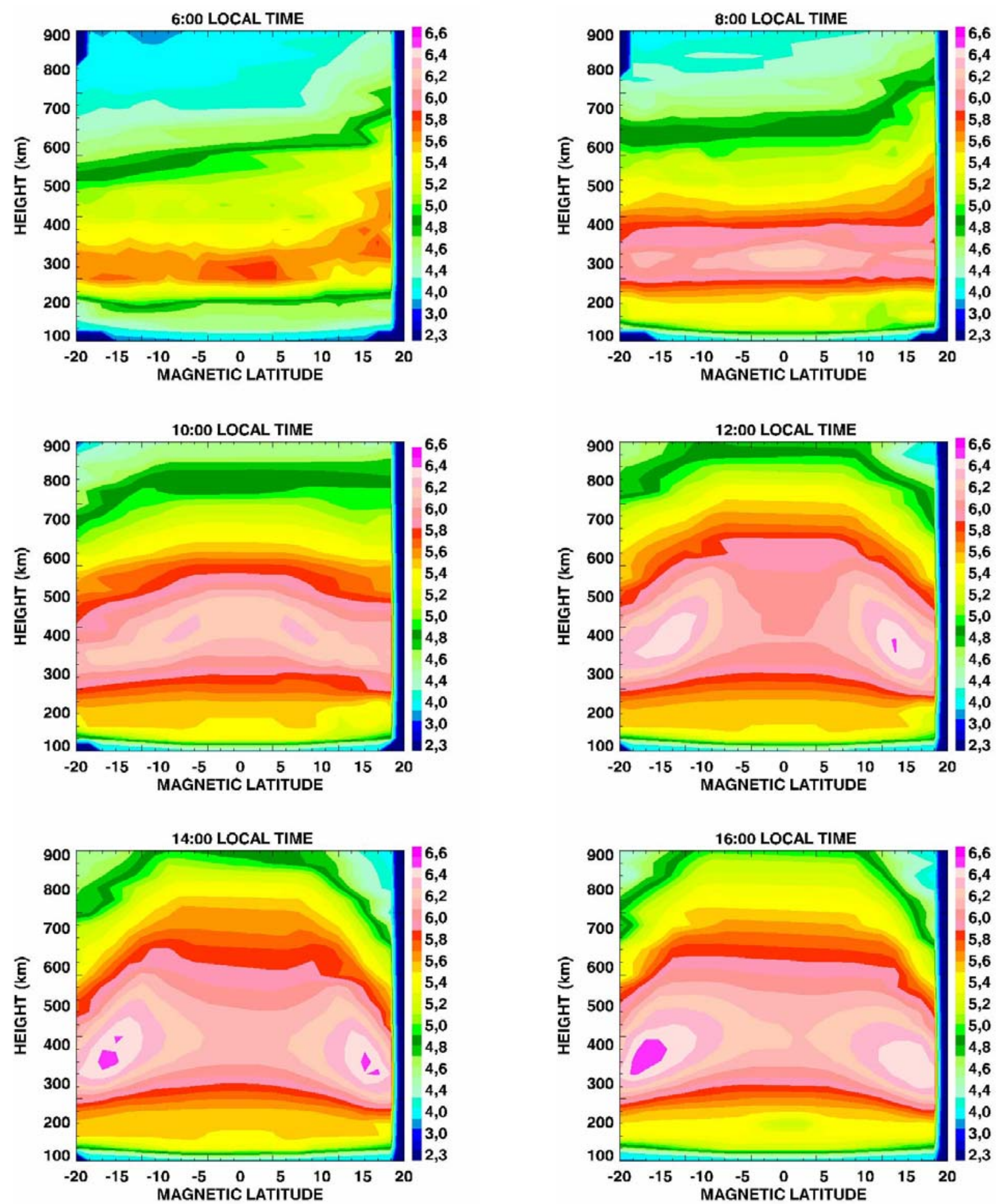

Fig. 3. Ionospheric electron density distribution as a function of height and magnetic latitude for various local times generated by the LION model for average solar conditions in the Brazilian longitudinal region, during equinox. 

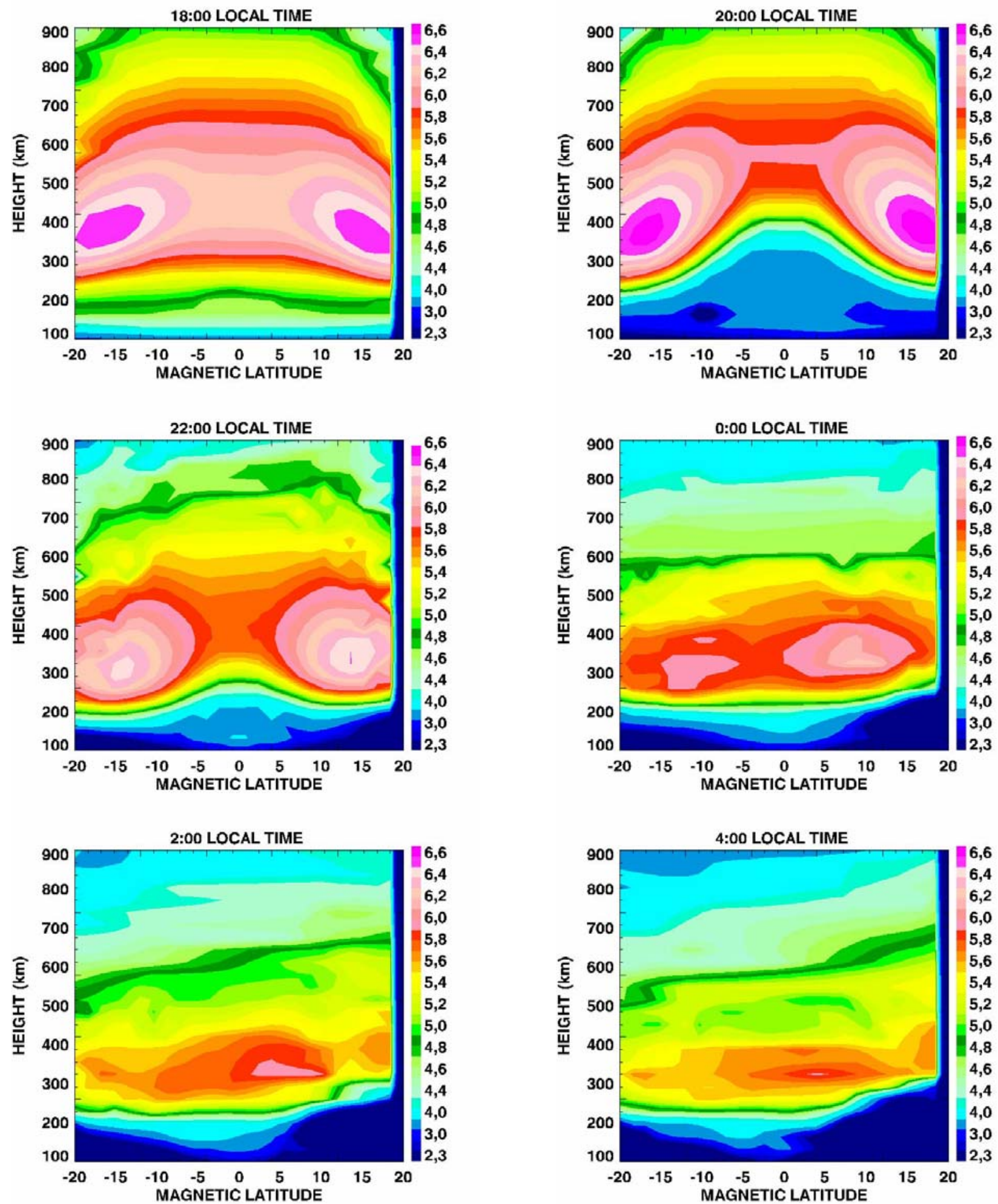

Fig. 3. Continued. 


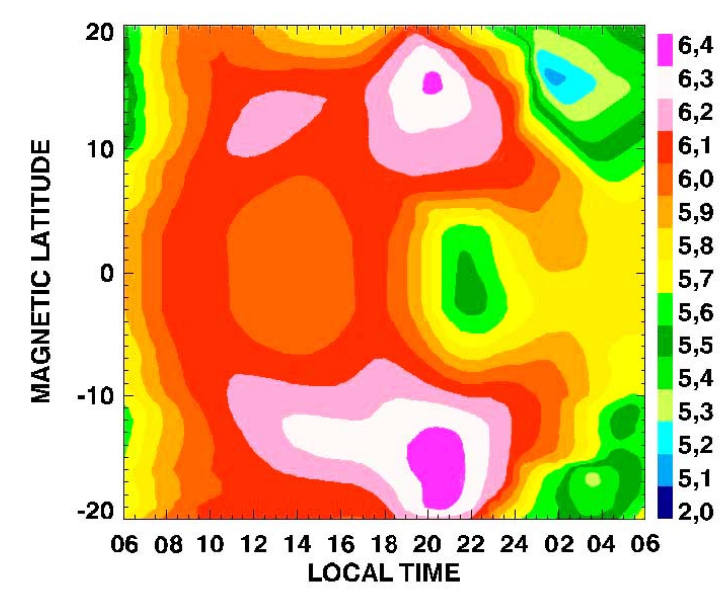

Fig. 4. Ionospheric electron density peak $\left(n_{m}\right)$ as a function of magnetic latitude and local time generated by the LION model for average solar conditions in the Brazilian longitudinal region, during summer in the Southern Hemisphere.

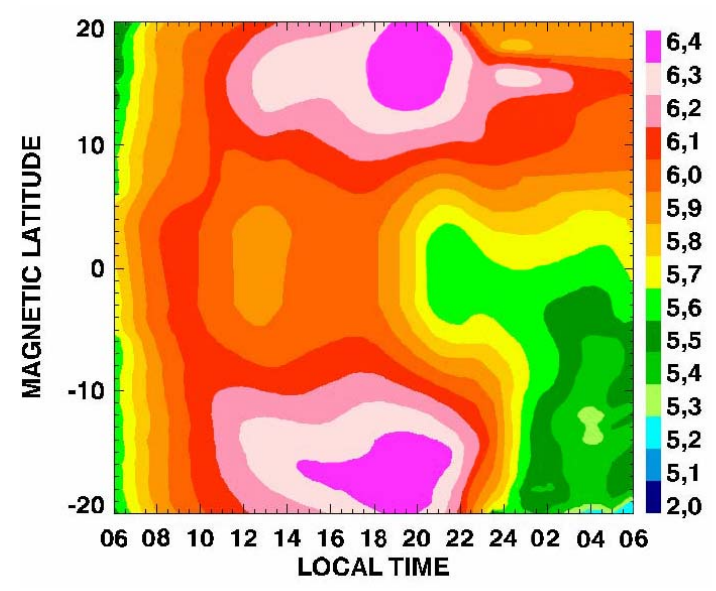

Fig. 5. Ionospheric electron density peak $\left(n_{m}\right)$ as a function of magnetic latitude and local time generated by the LION model for average solar conditions in the Brazilian longitudinal region, during equinox.

the pre-reversal enhancement) produces a more pronounced ionospheric anomaly in the early part of the night, and, in some cases (depending on other simultaneous conditions), is responsible for the generation of plasma irregularities and large scale plasma bubbles (depleted plasma regions) in the low latitude ionosphere, through plasma instability processes. When there are no vertical plasma drifts, the peak electron density maximizes near the magnetic equator in the late afternoon hours and the ionospheric equatorial anomaly is not generated. Also, because of the large electrical conductivity along the magnetic field lines, the vertical plasma drift, through the so-called fountain effect, produces a distribution of ionization that is symmetric about the magnetic equator, when a thermospheric neutral wind is not included.

One of the important effects of the thermospheric wind velocity along the magnetic field line is to produce a northsouth asymmetry in the ionization distribution about the magnetic equator. The neutral wind moves, through iondrag, the ionization upward in the upwind side of the magnetic field line, into regions where the recombination rate is lower, and downward in the downwind side of the magnetic field line, into regions where the recombination rate is higher, at the same time that it promotes an interhemisphere transport of ionization. Also, the peak height of the ionospheric F-layer is very sensitive to changes in the wind velocity direction so that when the there is a reversal in the wind velocity the north-south asymmetry in the F-layer peak height distribution is also reversed, showing a very fast response of the ionospheric peak height to changes in the wind direction. The same is not true for the electron density distribution due to the combined effects of recombination. In this sense, the height difference in the F2-region peak heights at conjugate magnetic latitudes, near the anomaly crests, can be used to infer the neutral wind velocities along the magnetic meridian (see, e.g. Bittencourt et al., 1976; Bittencourt and Sahai, 1978).

The north-south asymmetries in the ionization distribution, present in the color maps shown in Figs. 2, 3, 4 and 5 , are mainly due to thermospheric wind effects and, to a smaller extent, also due to neutral atmosphere asymmetries associated with the seasons. The asymmetries produced by the thermospheric winds are also strongly dependent on the magnetic declination and, therefore, on the longitudinal region considered. Depending on the season considered, and on the separation between geographic and magnetic equators, very strong thermospheric wind velocities can be responsible for large asymmetries in the ionization distribution and for fast decay (or not) of the ionization after sunset. Different wind models, as well as drift models, can generate different shapes of north-south asymmetries and ionization distributions as a function of latitude and local time.

Similar maps can also be produced for different solar conditions, different longitudes, and for various different geophysical parameters (such as plasma drift velocities, thermospheric wind velocities, ionization rates, collision frequencies, and so on), in order to analyze the ionospheric behavior under various circumstances.

\section{Summary and conclusions}

The low-latitude ionospheric (LION) computer model presented here generates the spatial distribution and time evolution of the number density and macroscopic velocity of the electrons and the ions $\mathrm{O}^{+}, \mathrm{O}_{2}^{+}, \mathrm{NO}^{+}, \mathrm{N}_{2}^{+}$and $\mathrm{N}^{+}$in the low-latitude ionosphere, considering various different geophysical and solar conditions. It permits the study of the ionospheric changes related to solar activity, including the 
solar cycle variation and changes due to geomagnetic storms, to seasonal and neutral atmosphere variations, to plasma dynamical processes such as electromagnetic plasma drift and thermospheric neutral wind coupling. Also, from the ion density distributions generated by the model, the intensity of various airglow emission lines due to recombination processes can be calculated as a function of space and time. These emissions constitute a powerful diagnostic technique to study, from the ground, various dynamical processes which occur in the low-latitude ionosphere. The total electron content along a given line of sight can be easily calculated from the ionization distribution generated by the model. The LION model results may also be used for studies related to electromagnetic wave propagation through the ionosphere, of great importance in GPS and telecommunications systems.

Different drift models can be used to represent different situations and conditions. As discussed earlier, the amplitude and duration of the pre-reversal peak in the upward plasma drift velocities is greatly dependent on the season and on the magnetic declination at a particular longitude. This dependence is due mainly to the variation in the low-latitude ionospheric conductivity at magnetically conjugate points near sunset as the terminator crosses the magnetic meridian, since the angle formed between these two lines depends on season and magnetic declination. The amplitude and duration of the pre-reversal peak will control the evolution of the equatorial anomaly in the hours just after sunset These effects can be included in the model through proper selection of the electromagnetic plasma drift velocities appropriate for each longitudinal sector and season.

Since the horizontal wind velocity that goes into the model is the wind component along the magnetic meridian, the effects of the geographic zonal and meridional wind components will depend on the value of magnetic declination at a specified longitude. According to Eq. (36), the effects of the geographic zonal wind component will be just opposite in the longitudinal regions where the magnetic declination is east or west (because of the sign of $\sin \delta_{m}$ ). Thus, the effect of the neutral wind on the low-latitude ionosphere is strongly longitudinal dependent, due to the longitudinal variation of magnetic declination. These effects can be included in the calculations through proper selection, for each longitudinal sector, of the wind velocity model $\left(u_{\theta}\right)$ along the magnetic meridian. The use of different wind models, as well as drift models, can generate different ionization distributions as a function of latitude and local time, and various shapes of north-south asymmetries.

Comparison of computer model results with ionospheric measurements and with existent empirical ionospheric models can provide important information for physical interpretation of ionospheric data and for improvement of empirical ionospheric models at low latitudes. We hope that in a subsequent paper we shall present a comparison between LION model results and ionospheric data obtained through ionosonde measurements in the Brazilian longitudinal region, as well as a comparison with empirical models such as the IRI model.

Topical Editor M. Pinnock thanks two anonymous referees for their help in evaluating this paper.

\section{References}

Abbur-Robb, M. F. K. and Windle, D. W.: On the day and night reversal in NmF2 north-south asymmetry, Planet. Space Sci., 17, 96-106, 1969.

Abdu, M. A.: Equatorial ionosphere-thermosphere system: Electrodynamics and irregularities, Adv. Space Res., 35, 771-787, 2005.

Appleton, E. V.: Two anomalies in the ionosphere, Nature, 157, 691-696, 1946.

Anderson, D. N.: A theoretical study of the ionospheric F-region equatorial anomaly, I. Theory, Planet. Space Sci., 21, 409-419, 1973a.

Anderson, D. N.: A theoretical study of the ionospheric F-region equatorial anomaly, II. Results in the American and Asian sectors, Planet. Space Sci., 21, 421-442, 1973 b.

Anderson, D. N., Mendillo, M., and Herniter, B.: A semiempirical low-latitude ionospheric model, Radio Sci., 22, 292-306, 1987.

Anderson, D. N., Forbes, J. M., and Codrescu, M.: A fully analytic low and middle latitude ionospheric model, J. Geophys. Res., 94(A2), 1520-1524, 1989.

Bailey, G. J. and Sellek, R.: A mathematical model of the Earth's plasmasphere and its application in a study of $\mathrm{He}^{+}$at $\mathrm{L}=3.0$, Ann. Geophys., 8, 171-190, 1990, http://www.ann-geophys.net/8/171/1990/.

Bailey, G. J., Sellek, R., and Rippeth, Y.: A modelling study of the equatorial topside ionosphere, Ann. Geophys., 11, 263-272, 1993, http://www.ann-geophys.net/11/263/1993/.

Banks, P. A. and Kockarts, G.: Aeronomy, Parts A and B, Academic Press, New York, USA, 1973.

Barth, C. A.: Nitric oxide in the upper atmosphere, Ann. Geophys., 22, 198-207, 1966, http://www.ann-geophys.net/22/198/1966/.

Bates, D. R.: Charge transfer and ion-atom interchange collisions, Proc. Phys. Soc. London, A68, 344-345, 1955.

Batista, I. S., Medeiros, R. T., Abdu, M. A., and Souza, J. R.: Equatorial ionospheric vertical plasma drift model over the Brazilian region, J. Geophys. Res., 101(A5), 10 887-10 892, 1996.

Batista, I. S., De Paula, E. R., Abdu, M. A., Trivedi, N. B., and Greenspan, M. E.: Ionospheric effects of the March 13, 1989, magnetic storm at low and equatorial latitudes, J. Geophys. Res., 96(A8), 13 943-13 952, 1991.

Batista, I. S., Abdu, M. A., and Medrano, R. A.: Magnetic activity effects on range type spread-F and vertical plasma drifts at Fortaleza and Huancayo as studied through ionosonde measurements and theoretical modeling, Ann. Geophys., 8, 357-364, 1990, http://www.ann-geophys.net/8/357/1990/.

Batista, I. S., Abdu, M. A., and Bittencourt, J. A.: Equatorial Fregion vertical plasma drifts: Seasonal and longitudinal asymmetries in the American sector, J. Geophys. Res., 91, 12055 12064, 1986. 
Bauer, S. J.: Physics of planetary ionospheres, Springer-Verlag, New York, USA, 1973.

Baxter, R. G.: A numerical solution of the time-varying diffusion equation for the F2-layer, Planet. Space Sci., 15, 701-713, 1967.

Baxter, R. G.: On the theoretical effects of electrodynamic drift in the equatorial F2-layer, J. Atmos. Terr. Phys., 711-720, 1964.

Baxter, R. G. and Kendall, P. C.: A theoretical technique for evaluating the time-dependent effects of general electrodynamic drifts in the F2-layer of the ionosphere, Proc. Royal Soc., A304, 171$185,1968$.

Biondi, M. A.: Atmospheric electron-ion and ion-ion recombination processes, Can J. Chem., 47, 1711-1719, 1969.

Bittencourt, J. A.: Fundamentals of plasma physics, SpringerVerlag, New York, USA, Third Edition, 2004.

Bittencourt, J. A.: The low-latitude ionosphere: A dynamic computer model, Research Report INPE-5965-RPQ/674, 1996.

Bittencourt, J. A. and Abdu, M. A.: A theoretical comparison between apparent and real vertical ionization drift velocities in the equatorial F-region, J. Geophys. Res., 86, 2451-2454, 1981.

Bittencourt, J. A. and Sahai, Y.: F-region neutral winds from ionosonde measurements of hmf 2 at low-latitude magnetic conjugate regions, J. Atmos. Terr. Phys., 40, 669-676, 1978.

Bittencourt, J. A., Sahai, Y., Teixeira, N. R., and Takahashi, H.: A comparative study of the low-latitude ionospheric and OI $630 \mathrm{~nm}$ nightglow observations with the SLIM and IRI models, Adv. Space Res., 12(6), 275-278, 1992.

Bittencourt, J. A. and Tinsley, B. A.: Tropical F-region winds from OI 1356 A and OI 6300 A emissions, 1. Theory, J. Geophys. Res., 81(22), 3781-3785, 1976.

Bittencourt, J. A., Tinsley, B. A., Hicks, G. T., and Reed, E. I.: Tropical F-region winds from OI $1356 \mathrm{~A}$ and OI $6300 \mathrm{~A}$ emissions. 2. Analysis of OGO 4 data, J. Geophys. Res., 81(22), 3786-3790, 1976.

Blum, P. W. and Harris, I.: The global wind system in the thermosphere, Space Research, XIII, 369-375, 1973.

Bramley, E. N. and Peart, M.: Diffusion and electromagnetic drift in the equatorial F2-region, J. Atmos. Terr. Phys., 27, 1201-1211, 1965.

Bramley, E. N. and Young, M.: Winds and electromagnetic drifts in the equatorial F2-region, J. Atmos. Terr. Phys., 30, 99-111, 1968.

Challinor, R. A.: Neutral air winds in the ionospheric F-region for an asymmetrical global pressure system, Planet. Space Sci., 18, 1485-1487, 1970.

Chapman, S. and Cowling, T.G.: The mathematical theory of nonuniform gases, Cambridge University Press, UK, 1939.

Chiu, Y. T.: An improved phenomenological model of ionospheric density, J. Atmos. Terr. Phys., 37, 1563-1570, 1975.

Crank, J. and Nicolson, P.: A practical method for numerical evaluation of solutions of partial differential equations of the heatconduction type, Cambridge Phil. Soc. Proc., 43, 50-67, 1947.

Dalgarno, A.: Ambipolar diffusion in the F-region, J. Atmos. Terr. Phys., 26, 939-954, 1964.

Danilov, A. D. and Morozova, L. D.: Ionospheric storms in the F2region. Morphology and Physics (Review), Geomag. Aeron., 25, 593-605, 1985.

Dalgarno, A.: Charged particles in the upper atmosphere, Ann. Geophys., 17, 16-49, 1961,

http://www.ann-geophys.net/17/16/1961/.
Donahue, T. M.: Ionospheric composition and reactions, Science, 159, 489-498, 1968.

Dunkin, D. B., Fehsenfeld, F. C., Schmeltekopf, A. L., and Ferguson, E. E.: Ion-molecule reaction studies from $300 \mathrm{~K}$ to $600 \mathrm{~K}$ in a temperature-controlled flowing afterglow system, J. Chem. Phys., 49, 1365-1371, 1968.

Dunkin, D. B., Mcfarland, M., Fehsenfeld, F. C., and Ferguson, E. E.: Rate constants for the reactions of $\mathrm{O}^{+}$with $\mathrm{NO}, \mathrm{N}_{2} \mathrm{O}$ and $\mathrm{NO}_{2}$, J. Geophys. Res., 76, 3820-3821, 1971.

Farley, D. T.: A theory of electrostatic fields in a horizontally stratified ionosphere subject to a vertical magnetic field, J. Geophys. Res., 64, 1225-1233, 1959.

Fehsenfeld, F. C., Dunkin, D. B., and Ferguson, E. E.: Rate constants for the reaction of $\mathrm{CO}_{2}^{+}$with $\mathrm{O}, \mathrm{O}_{2}$ and $\mathrm{NO} ; \mathrm{N}_{2}^{+}$with $\mathrm{O}$ and NO; and $\mathrm{O}_{2}^{+}$with NO, Planet. Space Sci., 18, 1267-1269, 1970.

Fejer, B. G., Souza, J. R., Santos, A. S., and Costa Pereira, A. E.: Climatology of F-region zonal plasma drifts over Jicamarca, J. Geophys. Res., 110, A12310, doi:10.1029/2005JA011324, 2005.

Fejer, B. G.: The electrodynamics of the low latitude ionosphere: Recent results and future challenges, J. Atmos. Solar-Terr. Phys., 59, 1465-1478, 1997.

Fejer, B. G., De Paula, E. R., Gonzalez, S. A., and Woodman, R. F.: Average vertical and zonal F-region plasma drifts over Jicamarca, J. Geophys. Res., 96, 13 901-13 906, 1991.

Fejer, B. G.: The equatorial ionospheric electric fields: A review, J. Atmos. Terr. Phys., 43, 377-386, 1991.

Fejer, B. G., De Paula, E. R., Batista, I. S., Bonelli, E., and Woodman, R. F.: Equatorial F-region vertical plasma drifts during solar maxima, J. Geophys. Res., 94, 12 049-12 054, 1989.

Fejer, B. G., Larsen, M. F., and Farley, D. T.: Equatorial disturbance dynamo electric fields, Geophys. Res. Lett., 10, 537-540, 1983.

Fejer, B. G.: Low-latitude electrodynamic plasma drifts: A review, J. Atmos. Terr. Phys., 53, 677-693, 1981.

Fejer, B. G., Gonzales, C. A., Farley, D. T., and Kelley, M. C.: Equatorial electric fields during magnetically disturbed conditions. 1. The effect of the interplanetary field, J. Geophys. Res., 84, 57975802, 1979.

Ferguson, E. E.: Laboratory measurements of F-region reaction rates, Ann. Geophys., 4, 819-823, 1969,

http://www.ann-geophys.net/4/819/1969/.

Ferguson, E. E.: Ionospheric ion-molecule reaction rates, Rev. Geophys., 5, 305-327, 1967.

Ferguson, E. E., Fehsenfeld, F. C., Goldan, P. D., and Schmeltekopf, A. L.: Positive ion-neutral reactions in the ionosphere, J. Geophys. Res., 70, 4323-4329, 1965.

Fuller-Rowell, T. J., Rees, D., Quegan S., Moffett, R. J., and Bailey, G. T.: Interactions between neutral thermospheric composition and the polar ionosphere using a coupled ionospherethermosphere model, J. Geophys. Res., 92, 7744-7748, 1987.

Fuller-Rowell, T. J. and Rees, D.: A three-dimensional timedependent global model of the thermosphere, J. Atmos. Sci., 37, 2545-2567, 1980.

Girard, A. and Petit, M.: Physics of the Earth's ionosphere, D. Reidel Pub. Comp., New York, USA, 1976.

Goldan, P. D., Schmeltekopf, A. L., Fehsenfeld, F. C., Schiff, H. I., and Ferguson, E. E., Thermal-energy ion-neutral reaction rates, 2. Some reactions of ionospheric interest, J. Chem. Phys., 44, 4095-4103, 1966. 
Hanson, W. B. and Moffett, R. J.: Ionization transport effects in the equatorial F-region, J. Geophys. Res., 71, 5559-5572, 1966.

Hargreaves, J. K.: The solar-terrestrial environment, Cambridge University Press, UK, 1992.

Hedin, A. E.: MSIS-86 thermospheric model, J. Geophys. Res., 92, 4649-4662, 1987.

Hedin, A. E., Biondi, M. A., Burnside, R. G., Hernandez, G., Johnson, R. M., Killeen, T. L., Mazaudier, C., Meriwether, J. W., Salah, J. E., Sica, R. J., Smith, R. W., Spencer, N. W., Wickwar, V. B., and Virdi, T. S.: Revised global model of thermosphere winds using satellite and ground-based observations, J. Geophys. Res., 96, 7657-7688, 1991.

Hedin, A. E., Spencer, N. W., and Killeen, T. L.: Empirical global model of upper thermosphere winds based on Atmosphere and Dynamics Explorer satellite data, J. Geophys. Res., 93, 99599978, 1988.

Heelis, R. A., Kendall, P. C., Moffett, R. J., Windle, D. W., and Rishbeth, H.: Electrical coupling of the $\mathrm{E}$ and $\mathrm{F}$ regions and its effects on F-region drifts and winds, Planet. Space Sci., 22(5), 743-756, 1974.

Hinteregger, H. E.: The extreme ultraviolet solar spectrum and its variation during a solar cycle, Ann. Geophys., 26, 547-554, 1970 http://www.ann-geophys.net/26/547/1970/

Hinteregger, H. E., Hall, L. A., and Schmidtke, G.: Solar XUV radiation and neutral particle distribution in the July 1963 thermosphere, Space Res., V, 1175-1190, 1965.

Huba, J. D., Joyce G., and Fedder, J. A.: Sami2 is another model of the ionosphere (SAMI2): A new low-latitude ionosphere model, J. Geophys. Res., 105, 23 035-23 044, 2000.

Jacchia, L. G.: Thermospheric temperature, density and composition: New models, Smiths. Astrophys. Obs. Spec. Rep., 375, 142, 1977.

Jacchia, L. G.: Revised static models of the thermosphere and exosphere with empirical temperature profiles, Smiths. Astrophys. Obs. Spec. Rep., 332, 1-48, 1971.

Jacchia, L. G.: Static diffusion models of the upper atmosphere with empirical temperature profiles, Smiths. Contrib. Astrophys. 8, 215-222, 1965.

Kasner, W. H., Biondi, M. A.: Electron-ion recombination in nitrogen, Phys. Rev., 137a, 317-329, 1965.

Kelley, M. C.: The Earth's ionosphere - Plasma physics and electrodynamics, Academic Press, San Diego, USA, 1989.

Kendall, P. C.: Geomagnetic control of diffusion in the F2-region of the ionosphere, I. The form of the diffusion operator, J. Atmos. Terr. Phys., 24, 805-811, 1962.

Keneshea, T. J., Narcisi, R. S., and Swider Jr., W.: Diurnal model of the E-region, J. Geophys. Res., 75, 845-854, 1970.

Kohl, H., and King, J. W.: Atmospheric winds between 100 and $700 \mathrm{~km}$ and their effects on the ionosphere, J. Atmos. Terr. Phys., 29, 1045-1062, 1967.

Lyon, A. J. and Thomas, L.: The F2-region equatorial anomaly in the African, American and East Asian sectors during sunspot maximum, J. Atmos. Terr. Phys., 25, 373-386, 1963.

Macnamara, L. F.: Ionosphere: Communications, surveillance and direction finding, Krieger Pub. Comp., Florida, USA, 1991

Martyn, D. F.: Electric currents in the ionosphere. III. Ionization drift due to winds and electric fields, Phil. Trans. Royal Soc., A246, 306-320, 1953.
Millward, G. H., Mueller-Wodarg, I. C. F., Aylward, A. D., FullerRowell, T. J., Richmond, A. D., and Moffett, R. J.: An investigation into the influence of tidal forcing on F-region equatorial vertical ion drift using a global ionosphere-thermosphere model with coupled electrodynamics, J. Geophys. Res., 106, 24733 $24744,2001$.

Moffett, R. J. and Hanson, W. B.: Effect of ionization transport on the equatorial F-region, Nature, 206, 705-706, 1965.

Norton, R. B.: The ionized constituents in the $100-300 \mathrm{~km}$ region of the Earth's upper atmosphere, Inst. Envir. Res. Tech. Mem. ITSA 60, U.S. Dept. Commerce, 1967.

Peymirat, C., Richmond, A. D., Emery, B. A., and Roble, R. G.: A magnetosphere-thermosphere- ionosphere electrodynamics general circulation model, J. Geophys. Res., 103(A8), $17467-$ 17477, 1998.

Piggott, R. and Rawer, K. (Eds.): U.R.S.I. handbook for ionogram interpretation and reduction, Rep. UAG-23, 2nd ed., p. 15, World Data Center A for Solar-Terrestrial Physics, Boulder, Colo., 1972.

Pillat, V. G.: Estudo da ionosfera em baixas latitudes através do modelo computacional LION e comparação com parâmetros ionosféricos observados, M. Sc. Thesis, UNIVAP, Brazil, 2006.

Pillat, V. G., Fagundes, P. R., and Bittencourt, J. A.: Ionospheric studies using a low-latitude ionospheric model (LION model) and ground-based ionosonde observations, in: 36 Committee on Space Research Scientific Assembly, 35 COSPAR, 2006, Pequim. Abstract Book, 2006.

Potter, D.: Computational physics, John Wiley \& Sons, Chinchester, UK, 1973, Reprinted 1980.

Rao, C. S. R.: Study of the geomagnetic anomaly during sunspot maximum, J. Atmos. Terr. Phys., 24, 729-737, 1962.

Rastogi, R. G.: The equatorial anomaly in the F2-region of the ionosphere, J. Inst. Telecom. Engrs., 12, 245-256, 1966.

Ratcliffe, J. A.: An introduction to the ionosphere and magnetosphere, Cambridge at the University Press, UK, 1972.

Richmond, A. D., Ridley, E. C., and Roble, R. G.: A thermosphereionosphere general circulation model with coupled electrodynamics, Geophys. Res. Lett., 19, 601-604, 1992.

Rishbeth, H.: Polarization fields produced by winds in the equatorial F-region, Planet. Space Sci., 19(3), 357-369, 1971.

Rishbeth, H.: Thermospheric winds and the F-region: A review, J. Atmos. Terr. Phys., 34, 1-47, 1972.

Rishbeth, H.: Dynamics of the equatorial F-region, J. Atmos. Terr. Phys., 39(9/10), 1159-1168, 1977.

Rishbeth, H.: The F-region dynamo, J. Atmos. Terr. Phys., 43(5/6), 387-391, 1981.

Rishbeht, H. and Garriot, O. K.: Introduction to ionospheric physics, Academic Press, New York, USA, 1969.

Roble, R. G. and Ridley, E. C.: A thermosphere-ionospheremesosphere-electrodynamics general circulation model (timeGCM): Equinox solar cycle minimum simulations $(30-500 \mathrm{~km})$, Geophys. Res. Lett., 21, 417-420, 1994.

Rush, C. M., Rush, S. V., Lyons, L. R., and Venkateswaran, S. V.: Equatorial anomaly during a period of declining solar activity, Radio Sci., 4, 829-841, 1969.

Scherliess, L. and Fejer, B. G.: Radar and satellite global equatorial F-region vertical drift model, J. Geophys. Res., 104, 6829-6842, 1999.

Schunk, R. W.: A mathematical model of the middle and high- 
latitude ionosphere, Pur. A. Geoph., 127, 255-303, 1988.

Schunk, R. W. and Walker, J. C. G.: Theoretical ion densities in the lower ionosphere, Planetary and Space Sciences, 21, 1875-1896, 1973.

Sojka, J. J.: Global scale, physical models of the F region ionosphere, Rev. Geophys., 27, 371-403, 1989.

Sterling, D. L., Hanson, W. B., and Woodman, R. F.: Synthesis of data obtained at Jicamarca, Peru, during the September 11, 1969, eclipse, Radio Sci., 7, 279-289, 1972.

Sterling, D. L., Hanson, W. B., Moffett, R. J., and Baxter, R. G.: Influence of electromagnetic drifts and neutral air winds on some features of the F2-region, Radio Sci., 4, 1005-1023, 1969.

Thomas, L.: The F2-region equatorial anomaly during solstice periods at sunspot maximum, J. Atmos. Terr. Phys., 30, 1631-1640, 1968.

Tinsley, B. A., Christensen A. B., Bittencourt, J. A., Gouveia, H., Angreji, P. D., and Takahashi, H.: Excitation of oxygen permitted line emissions in the tropical nightglow, J. Geophys. Res., 78, 1174-1186, 1973.
Tobiska, W. K.: Revised solar extreme ultraviolet flux model, J. Atmos. Terr. Phys., 53, 1005-1018, 1991.

Tobiska, W. K. and Barth, C. A.: A solar EUV flux model, J. Geophys. Res., 95(A6), 8243-8251, 1990.

Torr, D. G. and Torr, M. R.: Chemistry of the thermosphere and ionosphere, J. Atmos. Terr. Phys., 41, 797-839, 1979.

Walker, J. C. G.: Analytic representation of upper atmosphere densities based on Jacchia's static diffusion models, J. Atmos. Sci., 22, 462-463, 1965.

Whitten, R. C. and Poppoff, I. G.: Fundamentals of aeronomy, John Wiley \& Sons Inc., New York, USA, 1971.

Woodman, R. F.: East-west ionospheric drifts at the magnetic equator, Space Research, XII, 969-974, 1972.

Woodman, R. F.: Vertical drift velocities and east-west electric fields at the magnetic equator, J. Geophys. Res., 75, 6249-6259, 1970. 